\title{
PRODUCT-CONVOLUTION OPERATORS AND MIXED-NORM SPACES
}

BY

\author{
ROBERT C. BUSBY AND HARVEY A. SMITH
}

\begin{abstract}
Conditions for boundedness and compactness of product-convolution operators $g \rightarrow P_{h} C_{f} g=h \cdot(f * g)$ on spaces $L_{p}(G)$ are studied. It is necessary for boundedness to define a class of "mixed-norm" spaces $L_{(p, q)}(G)$ interpolating the $L_{p}(G)$ spaces in a natural way $\left(L_{(p p)}=L_{p}\right)$. It is then natural to study the operators acting between $L_{(p, q)}(G)$ spaces, where $G$ has a compact invariant neighborhood. The theory of $L_{(p, q)}(G)$ is developed and boundedness and compactness conditions of a nonclassical type are obtained. It is demonstrated that the results extend easily to a somewhat broader class of integral operators. Several known results are strengthened or extended as incidental consequences of the investigation.
\end{abstract}

1. Introduction. Convolution by $f$ in $L_{1}(R)$ defines a bounded operator, $C_{f}$, on $L_{p}(R)$ for $1 \leqslant p \leqslant \infty$; likewise, pointwise multiplication by $h$ in $L_{\infty}(R)$ defines a bounded operator $P_{h}$ on $L_{p}(R)$. Excepting trivial cases, these operators are never compact. The composition $P_{h} C_{f}$ of two such operators, which we term a productconvolution $(P C)$ operator, is frequently compact. Asking exactly when this occurs motivates this paper. $P C$ operators on $L_{p}$ of a locally compact group arise in many areas of analysis. In [2] and [3] the compactness of certain PC operators was used to study induced representations of locally compact groups. $P C$ operators (and their adjoint $C P$ operators) arise naturally in many applied problems.

Even with $h$ in $L_{\infty}(R)$ and $f$ in $L_{1}(R)$, we find that the "mixed-norm" spaces of [1] must be introduced to solve the compactness problem for $P_{h} C_{f}$. These spaces also arise unavoidably when one attempts to weaken conditions on $h$ and $f$ and keep $P_{h} C_{f}$ bounded. Various mixed-norm conditons on $h$ and $f$ guarantee boundedness of $P_{h} C_{f}$ from $L_{p}$ to $L_{q}$ and, in fact, between mixed-norm spaces. Conversely, $P_{h} C_{f}$ may be bounded from $L_{p}$ to $L_{q}$ (or from one mixed-norm space to another) with neither $h$ nor $f$ in any $L_{r}$, but both $h$ and $f$ must belong to mixed-norm spaces. Thus mixed-norm spaces are the natural setting for studying bounded $P C$ operators. We treat a wide class of $P C$ operators and, in all but one case, find necessary and sufficient conditions for compactness. The conditions involve only membership in a mixed-norm space or, in one instance, translational continuity in mixednorm spaces; they are usually easily checked for explicit examples. By applying various manipulations to $P C$ operators, we develop easily applied necessary and sufficient conditions for compactness of a wider variety of integral operators which

Received by the editors January 10, 1978 and, in revised form, January 11, 1980.

AMS (MOS) subject classifications (1970). Primary 43A15, 47B05; Secondary 22D15, 43A60. 
do not fall within the scope of classical compactness theorems. We attempt reasonable generality but do not pursue the extensions to vector-valued functions.

$\S 2$ establishes notation and reviews needed facts. In $\$ 3$ we define "uniform partitions" on locally compact groups and show they exist. We then use these partitions to define and study certain mixed-norm spaces. Many of these spaces on the real line have been studied by Holland [8], [9]. Kellogg [10] and Williams [17] studied analogous spaces on certain discrete groups. The paper [18] of Bertrandias, Datry, and Depuis appeared while this paper was in a referee's hands. It independently develops, for abelian groups $G$, spaces $l^{q}\left(L^{p}\right)$ equivalent to our mixed-norm spaces $L_{(p, q)}(G)$. We hoped in revision to use their definition, which cleverly avoids uniform partitions, but we found this obscured the mixed-norm structure and complicated our computations. Our techniques for nonabelian $G$ require the uniform partition and mixed-norm structure as do some spaces we use which are not equivalent to those of [18] even for abelian $G$. We have therefore not adopted the elegant approach of [18], which was developed for different ends.

The general construction of mixed-norm spaces as function spaces on products was given by Benedek and Panzone [1]. As a Borel space, $R$ is the product of an interval and the integers. The space $\underline{L}^{(p, q)}(I \times Z)$ of [1] consists of functions which are in $L_{p}([n, n+1])$ for each $n$ and such that the resulting sequence of $L_{p}(I)$ norms is in $l_{q}$. Emerson and Greenleaf [5] show every locally compact group to be the union of disjoint translates of some relatively compact Borel neighborhood. If the group is second countable it is borel isomorphic to the product of a discrete set with this neighborhood, so the construction on $R$ generalizes. Unfortunately this partition may not be conveniently related to group properties; moreover, we need arbitrarily fine partitions and it is not known that arbitrarily fine "EmersonGreenleaf neighborhoods" exist. These problems, rather than hesitancy to assume second countability, lead us to take an alternative approach in $\$ 3$.

Our $L_{(p, \infty)}$ seems a natural place to study almost periodic functions and stationary stochastic processes on $G$. (The Stepanovich a.p. functions are the closures in $L_{(p, \infty)}(R)$ of the trigonometric polynomials.) Particular mixed-norm spaces on $R^{n}$ studied by N. Wiener, R. Goldberg, and P. Szeptycki are cited in [18].

$\$ 4$ investigates convolution of functions from mixed-norm spaces and generalizes Young's inequality. In arbitrary locally compact groups, mixed-norm properties may be obscured by convolution. To prevent this, we assume the group is (IN), i.e., has a compact invariant neighborhood of the identity. This classical condition [6] is equivalent to existence of a nontrivial center in the group algebra [13]. (It seems unlikely (IN) is the weakest useful condition, but attempting to use unimodularity alone proved intractable.)

Our general Young's inequality shows the $L_{(p, 1)}(G)$ to be closed under convolution and hence a group algebra. It enables us to strengthen a result of Stewart [16] to show that there is always a bounded $L_{1}$ function on an abelian group having its Fourier transform of compact support and identically equal to one on a prescribed compact set. We also improve a result of Rickert [14], who showed that on a locally compact, noncompact group if $1 / p+1 / q<1$ there are $f$ in $L_{p}$ and $g$ in $L_{q}$ such 
that $f * g$ fails to exist on a set of positive measure. We give precise conditions on $f$ for this and show the set of such $f$ to be of second category in $L_{p}$.

In $\$ 5$ we consider the $P C$ operators which are bounded by amalgams of classical estimates and we show that if $P_{h} C_{f}$ is bounded $h$ and $f$ must belong to mixed-norm spaces.

In $\$ 6$ we give necessary and sufficient conditions for compactness of all the operators studied in $\$ 5$ with the exception of the case where the range space has infinite indices. (The difficulty there is lack of useable compactness criteria in $L_{\infty}$.) The conditions for compactness of $P_{h} C_{f}$ are mostly simply membership of $h$ and $f$ in ordinary mixed-norm spaces $L_{p, q}(G)$, but in one exceptional case a different space involving a "local-multiplier" condition must be used. The section concludes with a number of examples. $\$ 7$ summarizes our results with particular application to $L_{2}(R)$ and demonstrates the application to a wider class of operators.

2. Notation and preliminary remarks. We will always discuss a locally compact group, $G$. Functions are complex-valued and measurable unless otherwise specified. Measurability and integration is relative to a given left Haar measure $m$. If $1 \leqslant p \leqslant \infty$ then $p^{\prime}$ will denote the conjugate index: $1 / p+1 / p^{\prime}=1$. We denote the continuous complex-valued functions on $G$ by $C(G)$, while $C_{\infty}(G)$ and $K(G)$ denote functions vanishing at infinity and functions of compact support, respectively. For functions $f$ on $G$ we adopt the notations: $f^{\sim}(x)=f\left(x^{-1}\right),\left({ }_{y} f\right)(x)=$ $f(y x)$, and $f_{y}(x)=f(x y)$. By $f_{E}$ we always mean the product of $f \chi_{E}$ of $f$ by the characteristic function of a set $E$. If $E$ is Borel and of positive measure, there is automatic identification of $f_{E}$ with its restriction to $E$ which imbeds $L_{p}(E)$ (for the restricted Haar measure) in $L_{p}(G)$ and we make the identification routinely.

We will need pairs of extended real indices which we denote $\underline{v}=\left(v_{1}, v_{2}\right)$. Inequalities such as $\underline{v}<\underline{u}$ are interpreted termwise and, if $\boldsymbol{g}$ is a function of one variable, $g(v)$ means $\left(g\left(v_{1}\right), g\left(v_{2}\right)\right)$. If $r$ is a specific extended real number, we will denote the pair $(r, r)$ by $\bar{r}$. For any pair $\underline{v}$, we denote by $\underline{v}^{\prime}$ the conjugate pair defined by $1 / \underline{v}+1 / \underline{v}^{\prime}=\overline{1}$. We will use a partial ordering $>\operatorname{defined}$ by $\left(a_{1}, b_{1}\right)>$ $\left(a_{2}, b_{2}\right)$ iff $a_{1} \leqslant a_{2}$ and $b_{1} \geqslant b_{2}$. For any set $S$ of functions, we denote the nonnegative members by $S^{+}$.

We use the following repeatedly.

Proposition 2.1. Let $\left\{a_{1}, a_{2}, \ldots, a_{n}\right\}$ be nonnegative.

(a) If $1 \leqslant p<\infty$ and $1 / p+1 / q=1$, then

$$
\begin{aligned}
& n^{-1 / q}\left(a_{1}+a_{2}+\cdots+a_{n}\right) \leqslant\left(a_{1}^{p}+a_{2}^{p}+\cdots+a_{n}^{p}\right)^{1 / p}<\left(a_{1}+a_{2}+\cdots+a_{n}\right) . \\
& \text { (b) If } 0<p \leqslant 1 \text { and } 1 / p+1 / q=1 \text {, then } \\
& \left(a_{1}+a_{2}+\cdots+a_{n}\right) \leqslant\left(a_{1}^{p}+a_{2}^{p}+\cdots+a_{n}^{p}\right)^{1 / p} \leqslant n^{-1 / q}\left(a_{1}+a_{2}+\cdots+a_{n}\right) .
\end{aligned}
$$

Proof. (a) Apply Hölder's inequality and the monotonicity of norms to the measure space formed by assigning unit measure to the $\boldsymbol{n}$ integers.

(b) Apply (a) to $1 / p$ and the numbers $a_{i}^{p}$. 
3. Mixed norm spaces. To separate local and global properties of functions, we partition $G$ into subsets not too dissimilar in "size and shape".

Definition 3.1. Let $U, V$ be relatively compact open neighborhoods of the identity with $\bar{U} \subset V$. A partition $\pi$ of $G$ into disjoint Borel subsets is $U-V$ uniform if for each $E$ in $\pi$ there is $x$ such that $x U \subset E \subset x V$. A Borel partition is uniform if it is $U-V$ uniform for some $(U, V)$.

LEMMA 3.2. Let $F$ be a subset of $G$ such that for some fixed relatively compact open neighborhood $U$ of the identity $x U$ and $y U$ are disjoint whenever $x$ and $y$ are distinct members of $F$. For each pair $(K, L)$ of relatively compact Borel sets there is an integer $n_{F}(K, L) \geqslant 1$ such that each left translate of $L$ intersects at most $n_{F}(K, L)$ of the sets $x K$ for $x$ in $F$.

Proof. Let $n_{F}(K, L)$ be the greatest integer in the real number $m\left(L K^{-1} U\right) / m(U)$. If $x K \cap x^{\prime} L \neq \varnothing$ then $x \in x^{\prime} L K^{-1}$ so $x U \subset x^{\prime} L K^{-1} U$. Thus, if $x_{i} K$ intersect $x^{\prime} L$, for $x_{i}$ in $F(i=1,2, \ldots, n)$, the disjoint sets $x_{i} U$ are in $x^{\prime} L K^{-1}$, so $n m(U) \leqslant m\left(x^{\prime} L K^{-1} U\right)=m\left(L K^{-1} U\right)$. Hence $n \leqslant n_{F}(K, L)$.

We now show that uniform partitions exist in abundance.

Proposition 3.3. Let $U$ be a symmetric, open, relatively compact neighborhood of the identity. There exists a $U-U^{2}$ uniform partition of $G$.

Proof. Let $\mathcal{T}$ be a maximal family of pairwise disjoint left translates of $U$, $\mathcal{T}=\left\{x_{i} U_{i}: i \in I\right\}$ where $I$ is well-ordered with first element $i_{0}$. For any $x$, $x U \cap x_{i} U \neq \varnothing$ for some $i$, so $G=\cup_{i \in I} x_{i} U^{2}$. Let $W_{i_{0}}=\left(x_{i_{0}} U^{2}\right) \sim\left(\cup_{i \neq i_{0}} x_{i} U\right)$ (where $\sim$ denotes set difference). Since $x_{i_{0}} U^{2}$ meets only finitely many $x_{i} U$ by Lemma 3.2, $W_{i_{0}}$ is Borel. Suppose a Borel set $W_{i}$ has been defined for each $i<j$ such that

$\left(\alpha_{i}\right) \cup_{k \leqslant i} W_{k}=\left(\cup_{k \leqslant i} x_{k} U^{2}\right) \sim\left(\cup_{k>i} x_{k} U\right)$.

$\left(\beta_{i}\right)\left\{W_{k} \mid k \leqslant i\right\}$ are pairwise disjoint.

$\left(\gamma_{i}\right) W_{k} \subseteq x_{k} U^{2}$ for $k \leqslant i$.

We then define $W_{j}=\left(x_{j} U^{2}\right) \sim\left[\left(\cup_{i<j} W_{i}\right) \cup\left(\cup_{k>j} x_{k} U\right)\right]$. By Lemma 3.2, $x_{j} U^{2}$ meets only finitely many of the $W_{i}$ and the $x_{k} U$, so $W_{j}$ is Borel. Thus $W_{i}$ is defined by transfinite induction for all $i$ so that $\left(\alpha_{i}\right),\left(\beta_{i}\right)$ and $\left(\gamma_{i}\right)$ hold, but $\cup_{i \in I} W_{i}=$ $\cup_{i \in I} x_{i} U^{2}=G$ and $x_{i} U \subset W_{i} \subset x_{i} U^{2}$, so $\left\{W_{i}\right\}$ is a $U-U^{2}$ uniform partition.

Note that any $U-V$ uniform partition $\pi$ provides a situation in which Lemma 3.2 applies, since for each $E$ in $\pi$ we have $x_{E} U \subset E \subset x_{E} V$ and $F$ can be taken as one set of such $x_{E}$. The following results are immediate.

Proposition 3.4. Let $\pi$ and $F$ be as above, $K$ relatively compact and Borel. Every translate of $K$ intersects (and so is covered by) at most $n_{F}(V, K)$ members of $\pi$.

Proposition 3.5. Under the above conditions each left translate of a member of $\pi$ intersects at most $n_{F}(K, V)$ of the translates $x_{E} K$.

Definition 3.6. For any $p=\left(p_{1}, p_{2}\right), \overline{1} \leqslant p \leqslant \infty$, and uniform partition $\pi$, we say $f$ belongs to $L_{p}^{\pi}(G)$ if $\bar{f}_{E} \in L_{p_{1}}(E)$ for all $E$ in $\pi$ and the family of norms $\left\{\left\|f_{E}\right\|_{p_{1}}: E \in \pi\right\}$ belongs to $l_{p_{2}}(\pi)$. (We say $f$ is locally $L_{p_{1}}$ and globally $l_{p_{2}}$ relative to 
$\pi$. The $G$ is usually supressed and we write only $L_{p}^{\pi}$.) We define the norm $\|f\|_{p}^{\pi}$ to be the $l_{p_{2}}$ norm of $\left\{\left\|f_{E}\right\|_{p_{1}}: E \in \pi\right\}$. (It is easily seen that $L_{p}^{\pi}$ is a Banach space.)

We denote by $L_{\left(p_{1}, 0\right)}^{\pi}$ the closed subspace of $L_{\left(p_{1}, \infty\right)}^{\pi}$ consisting of functions locally in $L_{p_{1}}$ such that $\left\{\left\|f_{E}\right\|_{p_{1}}: E \in \pi\right\}$ vanishes at $\infty$ on $\pi$.

Proposition 3.7 (CF. [18, §7]). (a) For any $\pi$, and $1<p \leqslant \infty, L_{(p, p)}^{\pi}$ is identical (and isometric) with $L_{p}(G)$.

(b) If $\overline{1} \leqslant \underline{p} \leqslant \bar{\infty}, f \in L_{p}^{\pi}, g \in L_{p^{\prime}}^{\pi}$, then $f g \in L_{1}(G)$ and $\|f g\|_{1}<\|f\|_{p}^{\pi}\|g\|_{p^{\prime}}^{\pi}$. The mapping $\bar{F}_{g}: f \rightarrow \int_{G}|f g| d m$ is a bounded linear functional, and if $p<\bar{\infty}$ the

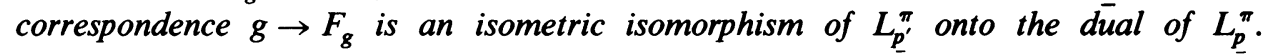
Similarly, the dual of $L_{(p, 0)}^{\pi}$ can be canonically identified with $L_{\left(p^{\prime}, 1\right)}^{\pi}$.

(c) If $\underline{p} \prec q$, then $L_{\underline{p}}^{\pi} \subset L_{q}^{\pi}$.

(d) If $1 \leqslant p \leqslant q \leqslant \infty$, then $L_{(p, q)}^{\pi} \supset L_{p}(G) \cup L_{q}(G)$ and $L_{(q, p)}^{\pi} \subset L_{p}(G) \cap$ $L_{q}(G)$.

(e) If $p<\bar{\infty}, K(G)$ is dense in $L_{p}^{\pi}$. Moreover, if $p_{1}<\infty, L_{\left(p_{1}, 0\right)}^{\pi}$ is the closure of $K(G)$ in $L_{\left(p_{1}, \infty\right)}^{\pi}$.

Proof. (a) is immediate from the definition, while (c) and (d) follow from the definition and elementary norm inequalities. Because the norms depend on $\pi$, there is some interest to the proof of (b). Applying Minkowski's inequality first on each $E$ in $\pi$ and then on the discrete set $\pi$, we have

$$
\int_{G}|f g| d m=\sum_{E \in \pi} \int_{E}\left|f_{E} g_{E}\right| d m \leqslant \sum_{E \in \pi}\left\|f_{E}\right\|_{p_{1}}\left\|g_{E}\right\|_{p_{1}^{\prime}} \leqslant\|f\|_{p_{-}}^{\pi}\|g\|_{p^{\prime}}^{\pi}
$$

which proves the first part of (b) and shows $F_{g}$ is in the dual of $L_{p}^{\pi}$.

If $\psi$ is in the dual of $L_{\underline{p}}{ }^{\pi}$ it restricts to a bounded linear functional $\psi_{E}$ on $L_{p_{1}}(E)$ for each $E$ in $\pi$. By the classical result, for some $g_{E}$ in $L_{p_{1}}(E),\left\|g_{E}\right\|_{p_{1}^{\prime}}=\left\|\psi_{E}\right\|$ and $\psi_{E}(f)=\int_{E} f g_{E} d m$ for all $f$ in $L_{p_{1}}(E)$. Let $g$ be the measurable function defined by setting $g(x)=g_{E}(x)$ for $x$ in $E$ and note that for $f$ in $L_{\underline{p}^{\prime}}, \psi(f)=\int_{G} f g d m$. We need only show $\|g\|_{p^{\prime}}^{\pi}=\|\psi\|$. Clearly $\|\psi\| \leqslant\|g\|_{p^{\prime}}^{\pi}<\infty$ by the first part of the proof. Given $\varepsilon>0$, choose, for each $E, k_{E}$ in $L_{p_{1}}(E)$ such that $\left\|k_{E}\right\|_{p_{1}}=1$ and $\psi_{E}\left(k_{E}\right)=\left|\psi_{E}\left(k_{E}\right)\right|>(1-\varepsilon)\left\|g_{E}\right\|_{p_{1}^{\prime}}$ For any function $\left\{\beta_{E}\right\}$ in $l_{p_{2}}$ of $\pi$, define $f(x)=\left|\beta_{E}\right| k_{E}(x)$ for $x$ in $E$, a member of $L_{\underline{p}}{ }^{\pi}$. Then

$$
\begin{aligned}
\|\psi\|\|\beta\|_{p_{2}} & =\|\psi\|\|f\|_{\bar{p}}^{\pi} \geqslant|\psi(f)|=\left|\sum_{E \in \pi}\right| \beta_{E}|| \psi_{E}\left(k_{E}\right)|| \\
& =\sum_{E \in \pi}\left|\beta_{E}\right|\left|\psi_{E}\left(k_{E}\right)\right| \geqslant(1-\varepsilon) \sum_{E \in \pi}\left|\beta_{E}\right|\left\|g_{E}\right\|_{p_{i}^{\prime}}
\end{aligned}
$$

Since $\beta$ is arbitrary in $l_{p_{2}}(\pi)$ it follows that $(1-\varepsilon)\left\|g_{E}\right\|_{p_{1}^{\prime}}$ is in $l_{p_{2}^{\prime}}(\pi)$ and has norm not greater than $\|\psi\|$. Since $\varepsilon$ is arbitrarily small, however, $\|g\|_{\underline{p}^{\prime}}^{\pi} \leqslant\|\psi\|$. The proof for $L_{\left(p_{1}, 0\right)}^{\pi}$ is similar.

(e) For any $f$ in $L_{\underline{p}}^{\pi}$ there is a finite $\alpha \subset \pi$ such that

$$
\left[\sum_{E \notin \alpha}\left(\left\|f_{E}\right\|_{p_{1}}\right)^{p_{2}}\right]^{1 / p_{2}}<\varepsilon / 2
$$

so, writing $F=\bigcup_{E \in \alpha} E,\left\|f-f_{F}\right\|_{\underline{p}}^{\pi}<\varepsilon / 2$. 
On the finite set $\alpha$ the $l_{p_{1}}$ and $l_{p_{2}}$ norms are equivalent since they are norms on the same finite-dimensional vector space, so on $F,\|\cdot\|_{\left(p_{1}, p_{2}\right)}^{\alpha}$ is equivalent to $\|\cdot\|_{\left(p_{1}, p_{1}\right)}^{\alpha}=\|\cdot\|_{p_{1}}$. Since $K(G)$ is dense in $L_{p_{1}}(G)$ it is also dense in $L_{p_{1}}(F)$ and we can find $g$ in $K(G)$ such that $\left\|f_{F}-g_{F}\right\|_{p_{1}}$ and hence

$$
\left\|f_{F}-g_{F}\right\|_{\left(p_{1}, p_{2}\right)}^{\alpha}=\left\|f_{F}-g_{F}\right\|_{\left(p_{1}, p_{2}\right)}^{\pi}
$$

is arbitrarily small.

Without loss of generality $g$ vanishes outside an open $H \supset F$ with $m(H \sim F)$ arbitrarily small. Recall we can find a closed $K \subset F$ and an open $H \supset F$ such that $m(H \sim K)$ is arbitrarily small and a continuous $h, h=1$ on $K, h=0$ on $G \sim H$, $0 \leqslant h \leqslant 1$. With $g$ above so that $\left\|f_{F}-g_{F}\right\|_{p_{1}}<\delta$, hg is in $K(G)$ and

$$
\left\|f_{F}-(h g)_{F}\right\|_{p_{1}} \leqslant\left\|f_{F}-g_{F}\right\|_{p_{1}}+\left\|g_{F}-(h g)_{F}\right\|_{p_{1}}<\delta+\max |g|(m(F-K))^{1 / p_{1}} .
$$

By choice of $K$ this is arbitrarily close to $\delta$ and $g$ can be replaced by $h g$. For $i=1,2,\left\|g-g_{F}\right\|_{p_{i}} \leqslant \max |g|(m(H-F))^{1 / p_{i}}$ can be made arbitrarily small by choice of $H$. If $p_{2} \geqslant p_{1}$, by the norm inequality for discrete spaces,

$$
\left\|g-g_{F}\right\|_{\left(., 1, p_{2}\right)}^{\pi} \leqslant\left\|g-g_{F}\right\|_{\left(p_{1}, p_{1}\right)}^{\pi}=\left\|g-g_{F}\right\|_{p_{1}} .
$$

If $p_{1} \leqslant p_{2}$, the reverse norm inequality, which holds for the finite measure spaces $E \in \pi$, yields (for some $C$ )

$$
\left\|g-g_{F}\right\|_{\left(p_{1}, p_{2}\right)}^{\pi} \leqslant C\left\|g-g_{F}\right\|_{\left(p_{2}, p_{2}\right)}^{\pi}=C\left\|g-g_{F}\right\|_{p_{2}} .
$$

Thus we can choose $g$ in $K(G)$ so that $\left\|f_{F}-g_{F}\right\|_{\left(p_{1}, p_{2}\right)}^{\pi}<\varepsilon / 4$ and $\left\|g-g_{F}\right\|_{\left(p_{1}, p_{2}\right)}^{\pi}<$ $\varepsilon / 4$ so $\left\|f_{F}-g\right\|_{\left(p_{1}, p_{2}\right)}^{\pi}<\varepsilon / 2$. Then $\|f-g\|_{\left(p_{1}, p_{2}\right)}^{\pi}<\left\|f-f_{F}\right\|_{\left(p_{1}, p_{2}\right)}^{\pi}+\left\|f_{F}-g\right\|_{\left(p_{1}, p_{2}\right)}^{\pi}$ $<\varepsilon$. Thus $K(G)$ is dense in $L_{\left(p_{1} p_{2}\right)}^{\pi}(G)$. The proof that it is dense in $L_{\left(p_{1}, 0\right)}^{\pi}(G)$ is similar.

We now show the norms $\|\cdot\|_{p}^{\pi}$ are equivalent as $\pi$ varies, so the topological vector spaces $L_{p}^{\pi}(G)$ are intrinsic to $G$.

Proposition 3.8. For $\pi^{\prime}$, $\pi$ uniform partitions, $\overline{1}<\underline{p} \leqslant \infty$, there is $M$ such that $\|f\|_{\underline{p}}^{\pi^{\prime}} \leqslant M\|f\|_{\underline{p}}^{\pi}$ for all measurable $f$.

Proof. For $E^{\prime} \in \pi^{\prime}$, let $S\left(E^{\prime}\right) \subset \pi$ be the set of $E \in \pi$ such that $E \cap E^{\prime} \neq \varnothing$. For $E \in \pi$, let $T(E) \subset \pi^{\prime}$ be the set of $E^{\prime}$ such that $E \in S\left(E^{\prime}\right)$. By 3.2 and corollaries, the cardinals of $S\left(E^{\prime}\right)$ and $T(E)$ have a common bound, $M$. Since $\left\|f_{E^{\prime}}\right\|_{p_{1}} \leqslant \sum_{E \in S\left(E^{\prime}\right)}\left\|f_{E}\right\|_{p_{1}}$ for all $E^{\prime}$ in $\pi^{\prime}$, it is immediate that $\|f\|_{\underline{p}}^{\pi^{\prime}}<M\|f\|_{p}^{\pi}$ if $p_{2}=\infty$. For $p_{2}<\infty, 2.1$ yields

so

$$
\left(\left\|f_{E^{\prime}}\right\|_{p_{1}}\right)^{p_{2}} \leqslant\left(\sum_{E \in S\left(E^{\prime}\right)}\left\|f_{E}\right\|_{p_{1}}\right)^{p_{2}} \leqslant M^{p_{2}-1} \sum_{E \in S\left(E^{\prime}\right)}\left(\left\|f_{E}\right\|_{p_{1}}\right)^{p_{2}}
$$

$$
\begin{aligned}
\|f\|_{\underline{p}}^{\pi^{\prime}} & \leqslant M^{\left(p_{2}-1\right) / p_{2}}\left(\sum_{E^{\prime} \in \pi^{\prime}} \sum_{E \in S\left(E^{\prime}\right)}\left(\left\|f_{E}\right\|_{p_{1}}\right)^{p_{2}}\right)^{1 / p_{2}} \\
& =M^{\left(p_{2}-1\right) / p_{2}}\left(\sum_{E^{\prime} \in T(E)} \sum_{E \in \pi}\left(\left\|f_{E}\right\|_{p_{1}}\right)^{p_{2}}\right)^{1 / p_{2}} \\
& \leqslant M\left(\sum_{E \in \pi}\left(\left\|f_{E}\right\|_{p_{1}}\right)^{p_{2}}\right)^{1 / p_{2}}=M\|f\|_{\underline{p}}^{\pi} .
\end{aligned}
$$


Next we give an intrinsic characterization of the spaces $L_{\underline{p}}^{\pi}$ and incidentally introduce new equivalent norms: $\left\|\left(|f|^{p_{1}} * g\right)^{1 / p_{1}}\right\|_{p_{2}}$.

Proposition 3.9. The following are pairwise equivalent for $1 \leqslant p_{1}<\infty$ :

(a) $f$ is in $L_{p}^{\pi}$.

(b) For some $g \neq 0$ in $K^{+}(G),\left(|f|^{p_{1}} * g\right)(x)$ exists for all $x$ in $G$ and $\left(|f|^{p_{1}} * g\right)^{1 / p_{1}}$ is in $L_{p_{2}}(G)$.

(c) For all $g$ in $K^{+}(G),\left(|f|^{p_{1}} * g\right)(x)$ exists for all $x$ in $G$ and $\left(|f|^{p_{1}} * g\right)^{1 / p_{1}}$ is in $L_{p_{2}}(G)$.

Proof. Clearly (c) implies (b). We show (a) implies (c). Let $\pi$ and $F$ be as in 3.4. Suppose $f \in L_{p}^{\pi}, g \in K^{+}(G), K$ the support of $g$ and $\beta=\max _{x}|g(x)|$. Let $T(E)=$ $\left\{E^{\prime} \in \pi: E^{\prime} \cap x_{E} V K^{-1} \neq \varnothing\right\}$ have cardinality $C_{1}(E)$ and $S(E)=\left\{E^{\prime} \in \pi: E \in\right.$ $\left.T\left(E^{\prime}\right)\right\}$ have cardinality $C_{2}(E)$. If $x \in E \in \pi$, then

$$
\begin{aligned}
\left(|f|^{p_{1}} * g\right)(x) & =\int_{G}|f(y)|^{p_{1}} g\left(y^{-1} x\right) d m(y) \leqslant \beta \int_{x_{E} V K^{-1}}|f(y)|^{p_{1}} d m(y) \\
& \leqslant \beta \sum_{E^{\prime} \in T(E)}\left(\left\|f_{E^{\prime}}\right\|_{p_{1}}\right)^{p_{1}}<\infty .
\end{aligned}
$$

By 3.2, $C_{1}(E) \leqslant n_{F}\left(V, V K^{-1}\right)$ so, for $1 \leqslant p_{2}<\infty, 2.1$ yields

$$
\begin{aligned}
{\left[\left(|f|^{p_{1}} * g\right)(x)\right]^{1 / p_{1}} } & \leqslant \beta^{1 / p_{1}} \sum_{E^{\prime} \in T(E)}\left\|f_{E^{\prime}}\right\|_{p_{1}} \\
& \leqslant\left(n_{F}\left(V, V K^{-1}\right)\right)^{\left(p_{2}-1\right) / p_{2}} \beta^{1 / p_{1}}\left[\sum_{E^{\prime} \in T(E)}\left(\left\|f_{E^{\prime}}\right\|_{p_{1}}\right)^{p_{2}}\right]^{1 / p_{2}} .
\end{aligned}
$$

Then

$$
\begin{aligned}
\int_{G}\left[\left(|f|^{p_{1}} * g\right)(x)\right]^{p_{2} / p_{1}} d m(x)=\sum_{E \in \pi} \int_{E}\left[\left(|f|^{p_{1}} * g\right)(x)\right]^{p_{2} / p_{1}} d m(x) \\
\leqslant m(V)\left(n_{F}\left(V, V K^{-1}\right)\right)^{\left(p_{2}-1\right)} \beta^{p_{2} / p_{1}} \sum_{E \in \pi} \sum_{E^{\prime} \in T(E)}\left(\left\|f_{E^{\prime}}\right\|_{p_{1}}\right)^{p_{2}} \\
=m(V)\left(n_{F}\left(V, V K^{-1}\right)\right)^{\left(p_{2}-1\right)} \beta^{p_{2} / p_{1}} \sum_{E \in S\left(E^{\prime}\right)} \sum_{E^{\prime} \in \pi}\left(\left\|f_{E^{\prime}}\right\|_{p_{1}}\right)^{p_{2}} \\
\leqslant m(V)\left(n_{F}\left(V, V K^{-1}\right)\right)^{\left(p_{2}-1\right)} \beta^{p_{2} / p_{1} n_{F}\left(V K^{-1}, V\right)\left(\|f\|_{\underline{P}}^{\pi}\right)^{p_{2}}<\infty}
\end{aligned}
$$

since $C_{2}\left(E^{\prime}\right) \leqslant n_{F}\left(V K^{-1}, V\right)$ by 3.5. Thus (a) implies (c). (The proof for $p_{2}=\infty$ is similar.)

Next we show (b) implies (a). (We show the more difficult case $p_{2}<\infty$.) Let $\pi$ and $F$ be as above, and let $g \neq 0$ in $K^{+}(G)$ be such that $\left(|f|^{p_{1 *}} g\right)^{1 / p_{1}}$ exists everywhere and lies in $L_{p_{2}}(G)$.

For some $\delta>0$, the open, relatively compact set $W=\{x: g(x)>\delta\} \neq \varnothing$, so, for all $x, f_{x W^{-1}}$ is in $L_{p_{1}}\left(x W^{-1}\right)$. By 3.5 , each $E^{\prime}$ in $\pi$ intersects only finitely many sets $x_{E} W^{-1}$, so $f$ is locally in $L_{p_{1}}$. Choose $y_{1}, y_{2}, \ldots, y_{k}$ such that $V^{-1} V \subseteq$ $\cup{ }_{j=1}^{k} y_{j} W^{-1}$. Then $\bar{g}=(1 / \delta) \sum_{j=1}^{k} g_{y_{j}} \in K^{+}(G)$. Since the hypothesis applies as well to right translates of $g$, we must have $\left(|f|^{p_{1}} * \bar{g}\right)^{1 / p_{1}}$ in $L_{p_{2}}(G)$. Choose $z_{E}$ in $E$ 
such that

$$
\begin{aligned}
m(E)^{-1} \int_{E}\left[\left(|f|^{p_{1}} * \bar{g}\right)(x)\right]^{p_{2} / p_{1}} d m(x) & \\
& \geqslant\left[\left(|f|^{p_{1}} * \bar{g}\right)\left(z_{E}\right)\right]^{p_{2} / p_{1}}=\delta^{-p_{2} / p_{1}}\left[\sum_{j=1}^{k}\left(|f|^{p_{1}} * g_{y_{j}}\right)\left(z_{E}\right)\right]^{p_{2} / p_{1}} \\
& \geqslant\left[\int_{S}|f(x)|^{p_{1}} d m(x)\right]^{p_{2} / p_{1}} \geqslant\left(\left\|f_{E}\right\|_{p_{1}}\right)^{p_{2}}
\end{aligned}
$$

where $S=\cup_{j=1}^{k} z_{E} y_{j} W^{-1} \supset z_{E} V^{-1} V \supset E$. Thus $m(U)^{-1 / p_{2}}\left\||f|^{p_{1}} * \bar{g}\right\|_{p_{2}} \geqslant$ $\|f\|_{p}^{\pi}$.

The proof of the following is analogous.

Proposition 3.10. The following are pairwise equivalent for $1 \leqslant p_{1}<\infty$.

(a) $f$ is in $L_{\left(p_{1}, 0\right)}^{\pi}$.

(b) $|f|^{p_{1}} * g$ is in $C_{\infty}(G)$ for some $g \neq 0$ in $K^{+}(G)$.

(c) $|f|^{p_{1}} * g$ is in $C_{\infty}(G)$ for all $g$ in $K^{+}(G)$.

The term "mixed-norm space" is used by Benedek and Panzone [1] for normed spaces of (equivalence classes of) functions on products of measure spaces. Our mixed norm spaces are equivalent to a special case of those defined by Benedek and Panzone. The next few propositions establish this, justifying our use of their term. We present them without proofs, which are straightforward.

Let $(X, \delta, v)$ and $(Y, \mathcal{T}, \rho)$ be totally $\sigma$-finite measure spaces. A measurable function $f$ on $(X \times Y, \delta \times \mathcal{T}, v \times \rho)$ is said in [1] to be in $\underline{L}^{p}(X \times Y)$ if $(\cdot, y)$ is in $L_{p_{1}}(X)$ for $(\rho)$ almost all $y$ in $Y$ and $\|f(\cdot, y)\|_{p_{1}}$ is in $L_{p_{2}}(Y)$. Then $\underline{L}^{p}$ is a Banach space in the obvious way.

Emerson and Greenleaf [5] prove the existence of a relatively compact Borel neighborhood $S$ of the identity and a discrete set $F \subset G$ such that $\pi=\{x S$ : $x \in F\}$ is a partition of $G$. (The countability hypothesis in the following is to avoid complications in defining product measures.)

Proposition 3.11. With the above notation, if $G$ is second countable it is Borel isomorphic with $S \times F$ in such a way that an isomorphic isomorphism is induced between $\underline{\underline{p}} \underline{\underline{p}}(S \times F)$ and $L_{\underline{p}}^{\pi}(G)$.

For $\overline{1} \leqslant \underline{p}<\bar{\infty}$ this shows $L_{\underline{p}}^{\pi}(G)$ is the norm completion of $L_{p_{1}}(S) \otimes l_{p_{2}}(F)$ which it contains in an obvious way.

We will now characterize compact subsets of $L_{\underline{p}}{ }^{\pi}$ for $\overline{1}<\underline{p}<\bar{\infty}$. We first show the set of translation operators is uniformly bounded on $L_{p}^{\pi}$.

LEMMA 3.12. Let $\pi$ and $F$ be as in 3.4. Then for $\overline{1}<\underline{p}<\infty$, if $f$ is in $L_{p}^{\pi}(G)$ then $\sup _{x \in G}\left\|_{x} f\right\|_{\underline{p}}^{\pi} \leqslant n_{F}(V, V)\|f\|_{\underline{p}}^{\pi}$.

Proof. Define $S(x E)=\left\{E^{\prime} \in \pi: x E \cap E^{\prime} \neq \varnothing\right\}, \quad T(x E)=\left\{E^{\prime} \in \pi: E \in\right.$ $\left.S\left(x E^{\prime}\right)\right\}$. By 3.4, neither $S(x E)$ nor $T(x E)$ has more than $n_{F}(V, V)$ elements, so by 
2.1 we have

$$
\begin{aligned}
& {\left[\int_{E}|x f(y)|^{p_{1}} d m(y)\right]^{p_{2} / p_{1}} } \leqslant\left[\sum_{E^{\prime} \in S(x E)} \int_{E^{\prime}}|f(y)|^{p_{1}} d m(y)\right]^{p_{2} / p_{1}} \\
& \leqslant {\left[\sum_{E^{\prime} \in S(x E)}\left[\int_{E^{\prime}}|f(y)|^{p_{1}} d m(y)\right]^{\left.1 / p_{1}\right]^{p_{2}}}\right.} \\
& \leqslant\left(n_{F}(V, V)\right)^{p_{2}-1} \sum_{E^{\prime} \in S(x E)}\left[\int_{E^{\prime}}|f(y)|^{p_{1}} d m(y)\right]^{p_{2} / p_{1}} ; \\
&\left(\|x\|_{p}^{\pi}\right)^{p_{2}} \leqslant\left[n_{F}(V, V)\right]^{p_{2}-1} \sum_{E \in \pi} \sum_{E^{\prime} \in S(x E)}\left[\int_{E^{\prime}}|f(y)|^{p_{1}} d m(y)\right]^{p_{2} / p_{1}} \\
&=\left[n_{F}(V, V)\right]^{p_{2}-1} \sum_{E^{\prime} \in \pi} \sum_{E \in T^{\prime}\left(x E^{\prime}\right)}\left[\int_{E^{\prime}}|f(y)|^{p_{1}} d m(y)\right]^{p_{2} / p_{1}} \\
& \leqslant\left[n_{F}(V, V)\right]^{p_{2}} \sum_{E^{\prime} \in \pi}\left[\int_{E^{\prime}}|f(y)|^{p_{1}} d m(y)\right]^{p_{2} / p_{1}}=\left[n_{F}(V, V)\right]^{p_{2}}\left(\|f\|_{\underline{p}}^{\pi}\right)^{p_{2}} .
\end{aligned}
$$

Proposition 3.13. For $\overline{1} \leqslant \underline{p}<\bar{\infty}$, a subset $H$ of $L_{\underline{p}}^{\pi}$ is relative compact iff

(a) $H$ is bounded.

(b) The family of maps of $G$ into $L_{\underline{p}}^{\pi}$ defined for each $f$ in $H$ by $x \rightarrow_{x} f$ is equicontinuous at the identity.

(c) For all $\varepsilon>0$, there is a set $J$ of compact complement such that $\left\|f_{J}\right\|_{\underline{p}}^{\pi}<\varepsilon$ for all $f$ in $H$.

Proof (Notation of 3.12). Necessity of (a) is clear by continuity of the norm. Let $k \in K(G)$ have support $S$. By 1.4 , at most $n_{F}(V, S)$ of $\pi$ intersect $S$, so

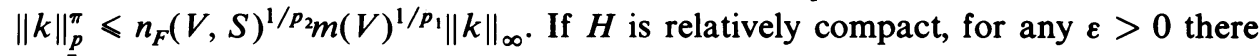
is a finite subset $\left\{k_{1}, k_{2}, \ldots, k_{n}\right\}$ of $K(G)$ such that, for any $f$ in $H,\left\|f-k_{i}\right\|_{p}^{\pi} \leqslant$ $\varepsilon / 2\left(n_{F}(V, V)+1\right)$ for some $i$. For $x$ in some neighborhood $W$ of the identity, we have $\left\|_{x} k_{i}-k_{i}\right\|_{p}^{\pi}<\varepsilon / 2$ for all $i$, so using 3.12 we have

$$
\begin{aligned}
\left\|{ }_{x} f-f\right\|_{\underline{p}}^{\pi} & \leqslant\left\|_{x} f-{ }_{x} k_{i}\right\|_{\underline{p}}^{\pi}+\left\|{ }_{x} k_{i}-k_{i}\right\|_{\underline{p}}^{\pi}+\left\|f-k_{i}\right\|_{\underline{p}}^{\pi} \\
& \leqslant\left[n_{F}(V, V)+1\right]\left\|f-k_{i}\right\|_{\underline{p}}^{\pi}+\left\|{ }_{x} k_{i}-k_{i}\right\|_{\underline{p}}^{\pi}<\varepsilon
\end{aligned}
$$

and (b) is proved. The necessity of (c) follows from choosing $\left\{k_{i}\right\}$ as above and noting that all the $k_{i}$ vanish outside a fixed compact set.

The proposition is known for $L_{p}(G)$. We use this to prove sufficiency. From (c), for any $\varepsilon>0$ we can find $Q=G \sim J$ such that, for $f$ in $H$,

$$
\left\|f_{J}\right\|_{\underline{p}}^{\pi}<\varepsilon / 2\left(n_{F}(V, V)+1\right) \text {. }
$$

From (b) we can find a neighborhood $W$ of the identity such that $\left\|_{x} f-f\right\|_{p}^{\pi}<\varepsilon / 2$ for all $x$ in $W$ and $f$ in $H$. From (a) we have $\left\|f_{Q}\right\|_{p}^{\pi}=\left\|f-f_{J}\right\|_{p}^{\pi}<\|f\|_{p}^{\pi}+\left\|f_{J}\right\|_{p}^{\pi}<$ $M+\varepsilon / 2$ for all $f$ in $H$. Consider the set $H_{Q}$ of all functions $f_{Q}, f$ in $\hat{H}$, as a subset of $L_{\underline{p}}{ }^{\pi}(Q)$. By our last remark, (a) applies to $H_{Q}$. But for $x$ in $W$ we have 


$$
\begin{aligned}
\left\|f_{x} f_{Q}-f_{Q}\right\|_{\underline{p}}^{\pi} & =\left\|\left({ }_{x} f-{ }_{x} f_{J}-\left(f-f_{J}\right)\right)\right\|_{\underline{p}}^{\pi} \leqslant\|\|_{x} f-f\left\|_{\underline{p}}^{\pi}+\right\| f_{x}-f_{J} \|_{\underline{p}}^{\pi} \\
& <\varepsilon / 2+\left\|f_{x}\right\|_{\underline{\underline{p}}}^{\pi}+\left\|f_{J}\right\|_{\underline{p}}^{\pi} \leqslant \varepsilon / 2+\left(n_{F}(V, V)+1\right)\left\|f_{J}\right\|_{\underline{p}}^{\pi}<\varepsilon .
\end{aligned}
$$

Thus (b) also applies to $H_{Q}$. By definition, (c) applies to the $H_{Q}$. On the compact set $Q$, however, the norm $\|\cdot\|_{p}^{\pi}$ and the usual $L_{p_{1}}(Q)$ norm are equivalent (by 2.1). Thus (a), (b), (c) hold for $H_{Q}$ regarded as a subset of $L_{p_{1}}(Q)$ and $H_{Q}$ is relatively compact in $L_{p_{1}}(Q)$. Thus we can find $\left\{h_{1}, h_{2}, \ldots, h_{n}\right\}$ in $H_{Q}$ such that for all $f$ in $H,\left\|f_{Q}-h_{i}\right\|_{p}^{\pi} \leqslant \varepsilon / 2$ for some $h_{i}$ (again, by norm equivalence). Thus $\left\|f-h_{i}\right\|_{p}^{\pi}=$ $\left\|f_{J}+f_{Q}-\bar{h}_{i}\right\|_{\underline{p}}^{\pi} \leqslant\left\|f_{J}\right\|_{\underline{p}}^{\pi}+\left\|f_{Q}-h_{i}\right\|_{\underline{p}}^{\pi}<\varepsilon$ and $H$ is totally bounded, hence relatively compact, in $L_{\underline{p}}{ }^{\pi}(G)$.

Our results on compactness of $P C$ operators require a mixed-norm space different from those discussed above. The remainder of the section develops these spaces.

Let $\Lambda_{U}$ denote the projection operator taking the measurable function $f$ to $f_{U}$ supported on the measurable set $U$ and let $T_{x}$ carry $f$ to $f_{x}$; note that $T_{x^{-1}} \Lambda_{U} T_{x}=$ $\Lambda_{U x}$.

Definition 3.14. For $1 \leqslant p, q \leqslant \infty$, we say $f$ is a local $p$ - $q$ multiplier if, for every pair $(E, H)$ of relatively compact sets of positive measure, $\Lambda_{H} C_{f} \Lambda_{E}: L_{p}(G) \rightarrow$ $L_{q}(G)$ (where $\left.\Lambda_{H} C_{f} \Lambda_{E}(g)=\left(f * g_{E}\right)_{H}\right)$ is defined and bounded. We denote the bound by $\left\|\Lambda_{H} C_{f} \Lambda_{E}\right\|_{p, q}$.

LEMMA 3.15. With the above notation, if $f$ is a local $p$-q multiplier where $1 \leqslant p$, $q<\infty$ then $\left\|\Lambda_{H} C_{f} \Lambda_{E}\right\|_{p, q}=\left\|\Lambda_{H x} C_{f} \Lambda_{E x}\right\|_{p, q}$.

Proof. $C_{f}$ commutes with right translation, so

$$
\Lambda_{H x} C_{f} \Lambda_{E x}=T_{x^{-1}} \Lambda_{H} T_{x} C_{f} T_{x^{-1}} \Lambda_{E} T_{x}=T_{x^{-1}} \Lambda_{H} C_{f} \Lambda_{E} T_{x}
$$

But writing $\Delta(x)$ for the modular function on $G$, we have

$$
\begin{gathered}
\left(\left\|T_{x^{-1}} \Lambda_{H} C_{f} \Lambda_{E} T_{x} h\right\|_{q}\right)^{q}=\int_{G}\left|\left(\Lambda_{H} C_{f} \Lambda_{E} T_{x} h\right)\left(y x^{-1}\right)\right|^{q} d m(y) \\
=\Delta(x) \int_{G}\left|\left(\Lambda_{H} C_{f} \Lambda_{E} T_{x} h\right)(u)\right|^{q} d m(u) \leqslant \Delta(x)\left(\left\|\Lambda_{H} C_{f} \Lambda_{E}\right\|_{p, q}\right)^{q}\left(\left\|T_{x} h\right\|_{p}\right)^{q} \\
=\Delta(x) \Delta\left(x^{-1}\right)\left(\left\|\Lambda_{H} C_{f} \Lambda_{E}\right\|_{p, q}\right)^{q}\left(\|h\|_{p}\right)^{q} .
\end{gathered}
$$

Thus $\left\|T_{x^{-1}} \Lambda_{H} C_{f} \Lambda_{E} T_{x}\right\|_{p, q} \leqslant\left\|\Lambda_{H} C_{f} \Lambda_{E}\right\|_{p, q}$ and symmetry, replacing $x$ by $x^{-1}$, yields equality. For the infinite index case, the same argument yields:

LeMMA 3.16. (a) $\left\|\Lambda_{H x} C_{f} \Lambda_{E x}\right\|_{\infty, \infty}=\left\|\Lambda_{H} C_{f} \Lambda_{E}\right\|_{\infty, \infty}$.

(b) $\left\|\Lambda_{H x} C_{f} \Lambda_{E x}\right\|_{p, \infty}=[\Delta(x)]^{-1 / p}\left\|\Lambda_{H} C_{f} \Lambda_{E}\right\|_{p, \infty}$ for $1<p<\infty$.

(c) $\left\|\Lambda_{H x} C_{f} \Lambda_{E x}\right\|_{\infty, q}=[\Delta(x)]^{1 / q}\left\|\Lambda_{H} C_{f} \Lambda_{E}\right\|_{\infty, q}$ for $1<q<\infty$.

An analog of Lemma 3.2 need not hold for right translates. There is no bound on the (finite) number of members of a uniform partition which can intersect a right translate of a member. This problem can be avoided if $G$ has a relatively compact invariant neighborhood of the identity, i.e., $G$ is an (IN) group. 
Definition 3.17. Let $G$ be an (IN) group, $\pi$ a uniform partition, and $1<p$, $q \leqslant \infty, 1 \leqslant s<\infty$. We say $f$ is a local $p-q$ multiplier of class $s$ relative to $\pi$ if, for some $H$ in $\pi, \Sigma_{E \in \pi}\left(\left\|\Lambda_{H} C_{f} \Lambda_{E}\right\|_{p, q}\right)^{s}<\infty$, i.e., $\left\|\Lambda_{H} C_{f} \Lambda_{E}\right\|_{p, q}$ (regarded as a function of $E$ in $\pi$ ) is in $l^{s}(\pi)$. We denote the space of such $f$ by $L M_{p, q ; s}^{\pi}$ and denote the norm in $l^{s}(\pi)$ by $\|f\|_{p, q ; s}^{\pi, H}$. Similarly $L M_{p, q ; \infty}^{\pi}$ and $L M_{p, q ; 0}^{\pi}$ are defined in terms of membership in $l^{\infty}(\pi)$ and $c_{0}(\pi)$, respectively.

Proposition 3.18. With the above notation, $L M_{p, q ; s}^{\pi}$ is independent of the choice of $H$; the norms obtained for different choices of $H$ are equivalent.

Proof. We give the case $1 \leqslant s<\infty$. Proofs for $s=0, \infty$ are similar. As usual, $\pi$ and $F$ are as in Proposition 3.4.

Let $N$ be a relatively compact invariant neighborhood of the identity, $H_{1}, H_{2}$ members of $\pi$. There is a finite set $A$ of cardinality $|A|$ such that $H_{2} \subset \cup_{x \in A} H_{1} x$. For $E \in \pi, x$ in $A$ we write

$$
\begin{gathered}
S(E, x)=\left\{E^{\prime} \in \pi: E^{\prime} \cap E x^{-1} \neq \varnothing\right\}, \\
T(E, x)=\left\{E^{\prime} \in \pi: E \in S\left(E^{\prime}, x\right)\right\} .
\end{gathered}
$$

There are $\left\{x_{1}, x_{2}, \ldots, x_{k}\right\}$ such that $E \subset \cup_{i} x_{i} N$ and if $E^{\prime} \in S(E, x)$ then $E^{\prime} \cap \cup_{i} x_{i} N x^{-1}=\cup_{i}\left(E^{\prime} \cap x_{i} x^{-1} N\right) \neq \varnothing$. By 3.4, $E^{\prime} \cap x_{i} x^{-1} N \neq \varnothing$ can hold for no more than $n_{F}(V, N)$ sets $E^{\prime}$ in $\pi$; the cardinality of $S(E, x)$ is at most $k n_{F}(V, N)$. Since every left translate of $V$ can be covered by the same number of left translates of $N, k$ can be independent of $E$. Thus $k n_{F}(V, N)$ is a bound on the cardinality of all the $S(E, x)$. A similar argument shows it is such a bound for the $T(E, x)$. Now

$$
\left\|\Lambda_{H_{2}} C_{f} \Lambda_{E}\right\|_{p, q} \leqslant \sum_{x \in A}\left\|\Lambda_{H_{1} x} C_{f} \Lambda_{E}\right\|_{p, q}=\sum_{x \in A}\left\|\Lambda_{H_{1}} C_{f} \Lambda_{E x^{-1}}\right\|_{p, q}
$$

by 3.15 and 3.16. (Recall that (IN) implies unimodular.) But

$$
\sum_{x \in A}\left\|\Lambda_{H_{1}} C_{f} \Lambda_{E x^{-1}}\right\|_{p, q} \leqslant \sum_{x \in A} \sum_{E^{\prime} \in S(E, x)}\left\|\Lambda_{H_{1}} C_{f} \Lambda_{E^{\prime}}\right\|_{p, q}
$$

since $\Lambda_{E x^{-1}}=\sum_{E^{\prime} \in S(E, x)} \Lambda_{E^{\prime}} \Lambda_{E x^{-1}}$. Thus, using 2.1,

$$
\begin{aligned}
\sum_{E \in \pi}\left(\left\|\Lambda_{H_{2}} C_{f} \Lambda_{E}\right\|_{p, q}\right)^{s} & \leqslant \sum_{E \in \pi}\left[\sum_{x \in A} \sum_{E^{\prime} \in S(E, x)}\left(\left\|\Lambda_{H_{1}} C_{f} \Lambda_{E^{\prime}}\right\|_{p, q}\right)\right]^{s} \\
& \leqslant \sum_{E \in \pi}\left[k n_{F}(V, N)|A|\right]^{s-1} \sum_{x \in A}\left[\sum_{E^{\prime} \in S(E, x)}\left(\left\|\Lambda_{H_{1}} C_{f} \Lambda_{E}\right\|_{p, q}\right)\right]^{s} \\
& =\sum_{E^{\prime} \in \pi} \sum_{x \in A} \sum_{E \in T\left(E^{\prime}, x\right)}\left[|A| k n_{F}(V, N)\right]^{s-1}\left(\left\|\Lambda_{H_{1}} C_{f} \Lambda_{E^{\prime}}\right\|_{p, q}\right)^{s} \\
& \leqslant\left(|A| k n_{F}(V, N)\right)^{s} \sum_{E^{\prime} \in \pi}\left(\left\|\Lambda_{H_{1}} C_{f} \Lambda_{E^{\prime}}\right\|_{p, q}\right)^{s}
\end{aligned}
$$

and by interchange of $H_{1}$ and $H_{2}$, the norms are equivalent and the spaces $L M_{p, q ; s}$ are independent of the choice of the set $H$, in their definition. Moreover, the equivalence class of norms is independent of $\pi$. 
4. Convolution and mixed-norm spaces. If $G$ is unimodular, $1 \leqslant p, q \leqslant \infty$, $1 / p+1 / q \geqslant 1$ and $1 / r=1 / p+1 / q-1$, then $L_{p}(G) * L_{q}(G) \subset L_{r}(G)$ and $\|f * g\|_{r} \leqslant\|f\|_{p}\|g\|_{q}$ (Young's inequality). Attempting to extend Young's inequality we encounter products of sets from $\pi$ and can find no bound on the number of members of $\pi$ intersecting such a product. As in the discussion of local multipliers, the appropriate assumption appears to be that $G$ is (IN). For the rest of this paper, $G$ will be an (IN) group and $N$ will denote a fixed symmetric compact invariant neighborhood of the identity.

LemMA 4.1. Let $\pi$ and $F$ be as in 3.4. For $E_{1}, E_{2}$ in $\pi$, define

$$
\begin{gathered}
S\left(E_{1}, E_{2}\right)=\left\{E \in \pi: E \cap E_{2} E_{1}^{-1} \neq \varnothing\right\}, \\
T\left(E_{1}, E_{2}\right)=\left\{E \in \pi: E_{1} \in S\left(E, E_{2}\right)\right\}, \\
W\left(E_{1}, E_{2}\right)=\left\{E \in \pi: E_{1} \in S\left(E_{2}, E\right)\right\} .
\end{gathered}
$$

There is an integer $\Gamma(\pi)$ which uniformly bounds $S, T$ and $W$.

Proof. Let $A$ be a finite set of cardinality $|A|$ such that $V \subset \cup_{y \in A} y N$. Then if $E \in S\left(E_{1}, E_{2}\right), \varnothing \neq x_{E} V \cap x_{E_{2}} V V^{-1} x_{E_{1}}^{-1} \subset \cup_{y \in A} \cup_{z \in A}\left[x_{E} V \cap x_{E_{2}} y z^{-1} x_{E_{1}}^{-1} N^{2}\right]$ by the invariance and symmetry of $N$ and $N^{2}$. By 3.2, there are at most $n_{F}\left(V, N^{2}\right) x_{E}$ for which each set of the union is nonempty. Thus $S\left(E_{1}, E_{2}\right)$ has at most $|A|^{2} n_{F}\left(V, N^{2}\right)$ members. Since $E \in T\left(E_{1}, E_{2}\right)$ (or $W\left(E_{1}, E_{2}\right)$ ) iff $E \cap$ $E_{1}^{-1} E_{2} \neq \varnothing$ (or $E \cap E_{1} E_{2} \neq \varnothing$ ), similar computations show we can choose $\Gamma(\pi)$ $=|A|^{2} n_{F}\left(V, N^{2}\right)$ in all cases.

TheOREM 4.2 (CF. [18]). Let $f \in L_{\underline{p}}^{\pi}, g \in L_{\underline{q}}^{\pi}, \overline{1} \leqslant \underline{p}, \underline{q} \leqslant \infty, 1 / p_{2}+1 / q_{2} \geqslant 1$; then

(a) $f * g$ exists (and is finite) locally a.e. and lies in $L_{\underline{r}}{ }^{\pi}$ where

$$
1 / r_{1}=\max \left\{0,1 / p_{1}+1 / q_{1}-1\right\}, \quad 1 / r_{2}=1 / p_{2}+1 / q_{2}-1 \text {. }
$$

(b) $\|f * g\|_{\underline{r}}^{\pi} \leqslant \Gamma(\pi)\|f\|_{\underline{p}}^{\pi}\|g\|_{\underline{q}}^{\pi}$.

Proof. Suppose $p_{2}, q_{2}, r_{2} \neq \infty$; then by Young's inequality

(i) $\left\|f_{E_{1}} * g_{E_{2}}\right\|_{r_{1}} \leqslant\left\|f_{E_{1}}\right\|_{p_{1}}\left\|g_{E_{2}}\right\|_{q_{1}}<\infty$ for any $E_{1}, E_{2}$ in $\pi$, and

(ii) $\left(f_{E_{1}} * g_{E_{2}}\right)_{E_{3}}=0$ if $E_{1} \notin S\left(E_{2}, E_{3}\right)$. Now

$$
\begin{gathered}
\sum_{E_{3} \in \pi} \sum_{E_{2} \in \pi} \sum_{E_{1} \in S\left(E_{2}, E_{3}\right)}\left(\left\|f_{E_{1}}\right\|_{p_{1}}\right)^{p_{2}}\left(\left\|g_{E_{2}}\right\|_{q_{1}}\right)^{q_{2}} \\
\quad=\sum_{E_{2} \in \pi}\left(\left\|g_{E_{2}}\right\|_{q_{1}}\right)^{q_{2}} \sum_{E_{1} \in \pi} \sum_{E_{3} \in U\left(E_{1}, E_{2}\right)}\left(\left\|f_{E_{1}}\right\|_{p_{1}}\right)^{p_{2}} \\
\leqslant \Gamma(\pi)\left(\|f\|_{p}^{\pi}\right)^{p_{2}}\left(\|g\|_{q}^{\pi}\right)^{q_{2}}<\infty .
\end{gathered}
$$

Thus for each $E_{3} \in \pi$,

$$
\sum_{E_{2} \in \pi} \sum_{E_{1} \in S\left(E_{2}, E_{3}\right)}\left(\left\|f_{E_{1}}\right\|_{p_{1}}\right)^{p_{2}}\left(\left\|g_{E_{2}}\right\|_{q_{1}}\right)^{q_{2}}<\infty
$$


By the generalized (multiproduct) Hölder inequality

$$
\begin{aligned}
& \sum_{E_{2} \in \pi} \sum_{E_{1} \in S\left(E_{2}, E_{3}\right)}\left(\left\|f_{E_{1}}\right\|_{p_{1}}\right)\left(\left\|g_{E_{2}}\right\|_{q_{1}}\right) \\
& \leqslant\left[\sum_{E_{2} \in \pi} \sum_{E_{1} \in S\left(E_{2}, E_{3}\right)}\left(\left\|f_{E_{1}}\right\|_{p_{1}}\right)^{p_{2}}\left(\left\|g_{E_{2}}\right\|_{q_{1}}\right)\right]^{1 / r_{2}} \\
& \cdot\left[\sum_{E_{2} \in \pi} \sum_{E_{1} \in S\left(E_{2}, E_{3}\right)}\left(\left\|f_{E_{1}}\right\|_{p_{1}}\right)^{p_{2}}\right]^{\left(q_{2}-1\right) / q_{2}} \\
& \cdot\left[\sum_{E_{2} \in \pi} \sum_{E_{1} \in S\left(E_{2}, E_{3}\right)}\left(\left\|g_{E_{2}}\right\|_{q_{1}}\right)^{q_{2}}\right]^{\left(p_{2}-1\right) / p_{2}} \\
& =\left[\sum_{E_{2} \in \pi} \sum_{E_{1} \in S\left(E_{2}, E_{3}\right)}\left(\left\|f_{E_{1}}\right\|_{p_{1}}\right)^{p_{2}}\left(\left\|g_{E_{2}}\right\|_{q_{1}}\right)^{q_{2}}\right]^{1 / r_{2}} \\
& \cdot\left[\sum_{E_{1} \in \pi} \sum_{E_{2} \in T\left(E_{1}, E_{3}\right)}\left(\left\|f_{E_{1}}\right\|_{p_{1}}\right)^{p_{2}}\right]^{\left(q_{2}-1\right) / q_{2}} \\
& \cdot\left[\sum_{E_{2} \in \pi} \sum_{E_{1} \in S\left(E_{2}, E_{3}\right)}\left(\left\|g_{E_{2}}\right\|_{q_{1}}\right)^{q_{2}}\right]^{\left(p_{2}-1\right) / p_{2}} \\
& \leqslant[\Gamma(\pi)]^{\left(2-1 / p_{2}-1 / q_{2}\right)}\left(\|f\|_{\underline{p}}^{\pi}\right)^{p_{2}\left(q_{2}-1\right) / q_{2}}\left(\|g\|_{\underline{q}}^{\pi}\right)^{q_{2}\left(p_{2}-1\right) / p_{2}} \\
& \cdot\left[\sum_{E_{2} \in \pi} \sum_{E_{1} \in S\left(E_{2}, E_{3}\right)}\left(\left\|f_{E_{1}}\right\|_{p_{1}}\right)^{p_{2}}\left(\left\|g_{E_{2}}\right\|_{q_{1}}\right)^{q_{2}}\right]^{1 / r_{2}} .
\end{aligned}
$$

Thus for any $E_{3}$, by (ii)

$$
\begin{aligned}
\left(\left\|(f * g)_{E_{3}}\right\|_{r_{1}}\right)^{r_{2}} \leqslant & {\left[\sum_{E_{2} \in \pi} \sum_{E_{1} \in S\left(E_{2}, E_{3}\right)}\left\|f_{E_{1}} * g_{E_{2}}\right\|_{r_{1}}\right]^{r_{2}} } \\
\leqslant & {[\Gamma(\pi)]^{\left(2-1 / p_{2}-1 / q_{2}\right) r_{2}}\left(\|f\|_{\underline{p}}^{\pi}\right)^{r_{2} p_{2}\left(q_{2}-1\right) / q_{2}}\left(\|g\|_{\underline{q}}^{\pi}\right)^{r_{2} q_{2}\left(p_{2}-1\right) / p_{2}} } \\
& \cdot\left[\sum_{E_{2} \in \pi} \sum_{E_{1} \in S\left(E_{2}, E_{3}\right)}\left(\left\|f_{E_{1}}\right\|_{p_{1}}\right)^{p_{2}}\left(\left\|g_{E_{2}}\right\|_{q_{1}}\right)^{q_{2}}\right], \\
\left(\|f * g\|_{\underline{r}}^{\pi}\right)^{r_{2}=} & \sum_{E_{3} \in \pi}\left(\left\|(f * g)_{E_{3}}\right\|_{r_{1}}\right)^{r_{2}} \\
\leqslant & {[\Gamma(\pi)]^{\left(2-1 / p_{2}-1 / q_{2}\right) r_{2}}\left(\|f\|_{\underline{p}}^{\pi}\right)^{r_{2} p_{2}\left(q_{2}-1\right) / q_{2}}\left(\|g\|_{q}^{\pi}\right)^{r_{2} q_{2}\left(p_{2}-1\right) / p_{2}} } \\
& \cdot\left[\sum_{E_{3} \in \pi \quad \sum_{E_{2} \in \pi} E_{1} \in S\left(E_{2}, E_{3}\right)}\left(\left\|f_{E_{1}}\right\|_{p_{1}}\right)^{p_{2}}\left(\left\|g_{E_{2}}\right\|_{q_{1}}\right)\right]^{q_{2}} \\
\leqslant & {[\Gamma(\pi)]^{\left(2-1 / p_{2}-1 / q_{2}\right) r_{2}+1}\left(\|f\|_{\underline{p}}^{\pi}\right)^{p_{2}+r_{2} p_{2}\left(q_{2}-1\right) / q_{2}}\left(\|g\|_{\underline{q}}^{\pi}\right)^{q_{2}-r_{2} q_{2}\left(p_{2}-1\right) / p_{2}} } \\
= & {[\Gamma(\pi)]^{r_{2}}\left(\|f\|_{\underline{p}}^{\pi}\right)^{r_{2}}\left(\|g\|_{q}^{\pi}\right)^{r_{2}}, }
\end{aligned}
$$

which proves both (a) and (b) for this case. 
Suppose $p_{2}, q_{2} \neq \infty$ but $r_{2}=\infty$ (so $1 / p_{2}+1 / q_{2}=1$ ). By 4.1 and 2.1

$$
\begin{aligned}
\sum_{E_{2} \in \pi}\left(\sum_{E_{1} \in S\left(E_{2}, E_{3}\right)}\left\|f_{E_{1}}\right\|_{p_{1}}\right)^{p_{2}} & \leqslant[\Gamma(\pi)]^{p_{2} / q_{2}} \sum_{E_{2} \in \pi} \sum_{E_{1} \in S\left(E_{2}, E_{3}\right)}\left(\left\|f_{E_{1}}\right\|_{p_{1}}\right)^{p_{2}} \\
& =[\Gamma(\pi)]^{p_{2} / q_{2}} \sum_{E_{1} \in \pi} \sum_{E_{2} \in T\left(E_{1}, E_{3}\right)}\left(\left\|f_{E_{1}}\right\|_{p_{1}}\right)^{p_{2}} \\
& \leqslant[\Gamma(\pi)]^{\left(p_{2} / q_{2}\right)+1} \sum_{E_{1} \in \pi}\left(\left\|f_{E_{1}}\right\|_{p_{1}}\right)^{p_{2}} \\
& =[\Gamma(\pi)]^{p_{2}}\left(\|f\|_{\underline{p}}^{\pi}\right)^{p_{2}} .
\end{aligned}
$$

Computing as before

$$
\begin{aligned}
\left\|(f * h)_{E_{3}}\right\|_{r_{1}} & \leqslant \sum_{E_{2} \in \pi} \sum_{E_{1} \in S\left(E_{2}, E_{3}\right)}\left\|f_{E_{1}}\right\|_{p_{1}}\left\|g_{E_{2}}\right\|_{q_{1}} \\
& =\sum_{E_{2} \in \pi}\left\|g_{E_{2}}\right\|_{q_{1}} \sum_{E_{1} \in S\left(E_{2}, E_{3}\right)}\left\|f_{E_{1}}\right\|_{p_{1}} \\
& \leqslant\left(\sum_{E_{2} \in \pi}\left(\left\|g_{E_{2}}\right\|_{q_{1}}\right)^{q_{2}}\right)^{1 / q_{2}}\left(\sum_{E_{2} \in \pi}\left(\sum_{E_{1} \in S\left(E_{2}, E_{3}\right)}\left\|f_{E_{1}}\right\|_{p_{1}}\right)^{p_{2}}\right)^{1 / p_{2}} \\
& \leqslant \Gamma(\pi)\|f\|_{\underline{m}}^{\pi}\|g\|_{\underline{q}}^{\pi} .
\end{aligned}
$$

Thus for this case we also have $\|f * g\|_{\underline{r}}^{\pi}=\sup _{E_{3} \in \pi}\left\|(f * g)_{E_{3}}\right\|_{r_{1}}<\Gamma(\pi)\|f\|_{\underline{p}}^{\pi}\|g\|_{\underline{q}}^{\pi}$.

Now suppose $p_{2}=\infty$ (so $q_{2}=1, r_{2}=\infty$ ). Then $\sup _{E_{1} \in \pi}\left\|f_{E_{1}}\right\|_{p_{1}}^{-}<\infty$, $\Sigma_{E_{2} \in \pi}\left\|g_{E_{2}}\right\|_{q_{1}}<\infty$

$$
\begin{aligned}
\left\|(f * g)_{E_{3}}\right\|_{r_{1}} & \leqslant \sum_{E_{2} \in \pi} \sum_{E_{1} \in S\left(E_{1}, E_{2}\right)}\left\|f_{E_{1}}\right\|_{p_{1}}\left\|g_{E_{2}}\right\|_{q_{1}} \leqslant\|f\|_{\underline{p}}^{\pi} \Gamma(\pi) \sum_{E_{2} \in \pi}\left\|g_{E_{2}}\right\|_{q_{1}}, \\
\|f * g\|_{\underline{r}}^{\pi} & \leqslant \Gamma(\pi)\|f\|_{\underline{p}}^{\pi}\|g\|_{\underline{g}}^{\pi} .
\end{aligned}
$$

A similar calculation yields the case $q_{2}=\infty$.

REMARKs. (i) By 3.13(b) translation is continuous, so from $4.2 f * g$ is uniformly continuous if $f \in L_{\underline{p}}^{\pi}, g \in L_{p^{\prime}}^{\pi}, \overline{1}<p<<$, just as for usual $L_{p}$ 's.

(ii) It follows from 4.2 that the $\bar{L}_{(p, 1)}^{\pi}(G)$ are group algebras (i.e., closed under convolution) for all $p \geqslant 1$.

(iii) J. Stewart has proved [16, §4, Lemma 1] for $G$ abelian with dual $G^{\wedge}$, that for every compact set $K$ in $G^{n}$, there is $f$ in $L_{(2,1)}^{\pi}(G) \cap L_{1}(G)$ such that its Fourier transform $f^{\prime} \equiv 1$ on $K$ and has compact support. (By 3.7(c) we know $L_{(2,1)}^{\pi} \subset L_{1}$, so Stewart's condition is redundant.) By 4.2, $f * f$ is in $L_{(\infty, 1)}^{\pi}(G) \subset L_{1}(G) \cap L_{\infty}(G)$ and its Fourier transform $\left(f^{2}\right)^{2}$ has the desired properties. Thus there is actually a bounded $L_{1}$ function with such a Fourier transform.

Lemma 4.3. Suppose $1 \leqslant p<\infty$. For every nonnegative sequence $\alpha$ not in $l_{p}\left(Z^{+}\right)$ there is a nonnegative sequence $\beta$ in $l_{p^{\prime}}\left(Z^{+}\right)$such that $\sum_{n=1}^{\infty} \alpha_{n} \beta_{n}=+\infty$.

Proof. If $p=1$, set $\beta_{n} \equiv 1$. If $p=\infty$, choose a subsequence such that $\alpha_{k}>k$. Let the corresponding $\beta_{k}$ be $1 / k^{2}$ and let $\beta$ vanish elsewhere. Then clearly $\beta$ is in $l_{1}\left(Z^{+}\right)$and the condition holds. Finally, suppose $1<p<\infty$. By assumption, 
$\sum_{n=1}^{\infty}\left(\alpha_{n}\right)^{p}=+\infty$, so if $D_{n}=\left(\alpha_{1}\right)^{p}+\left(\alpha_{2}\right)^{p}+\cdots+\left(\alpha_{n}\right)^{p}$, Dini's theorem on sequences (see [11]) says $\sum_{n=1}^{\infty}\left(\alpha_{n}\right)^{p} /\left(D_{n}\right)^{\gamma}$ is infinite for $\gamma \leqslant 1$ and finite for $\gamma>1$. Let $\beta_{n}=\alpha_{n}^{p-1} / D_{n}$. Then by Dini's theorem

$$
\begin{aligned}
\sum_{n=1}^{\infty} \beta_{n}^{p^{\prime}} & =\sum_{n=1}^{\infty} \frac{\alpha_{n}^{(p-1) p^{\prime}}}{D_{n}^{p^{\prime}}}=\sum_{n=1}^{\infty} \frac{\alpha_{n}^{p}}{D_{n}^{p^{\prime}}}<\infty, \\
\sum_{n=1}^{\infty} \alpha_{n} \beta_{n} & =\sum_{n=1}^{\infty} \frac{\alpha_{n}^{p}}{D^{n}}=+\infty
\end{aligned}
$$

THEOREM 4.4. For $f$ locally measurable and $\overline{1} \leqslant p \leqslant \infty$ the following are equivalent.

(a) $(f * g)$ exists (and is finite) locally a.e. for all $g$ in $L_{p}^{\pi}$.

(b) $(g * f)$ exists (and is finite) locally a.e. for all $g$ in $^{-} L_{p}{ }^{\pi}$.

(c) $f$ is in $L_{\left(1, p_{2}^{\prime}\right)}^{\pi}$.

Proof. By 3.8, we can assume $\pi$ in $N-N^{2}$ uniform. By symmetry and invariance of $N, \pi^{-1}=\left\{E^{-1}: E \in \pi\right\}$ is also an $N-N^{2}$ uniform partition. By 3.8, the map $f \rightarrow f^{\sim}, f^{\sim}(x)=f\left(x^{-1}\right)$, leaves all the spaces $L_{\underline{p}}^{\pi}$ invariant. Since $f * g=$ $\left(g^{\sim} * f^{\sim}\right) \sim$ it follows that (a) and (b) are equivalent.

By 4.2, (c) implies (a) and, in fact, $f * g$ is in $L_{\left(p_{1}, \infty\right)}^{\pi}$. We need only prove (a) implies (c). Let $E$ be in $\pi, W$ a relatively compact neighborhood of the identity, and $g$ the characteristic function of $E^{-1} W^{-1}$. Since $g \in L_{\underline{p}}{ }^{\pi}$, (a) implies that $f * g$ exists for some $x$ in $W^{-1}$;

$$
\infty>\int_{G}|f(y)| g\left(y^{-1} x\right) d m(y)=\int_{x W E}|f(y)| d m(y) \geqslant \int_{E}|f(y)| d m(y) ;
$$

$f$ is locally integrable.

Choose a maximal family of pairwise disjoint left translates $\left\{y_{j} N^{2}: j \in J\right\}$ of $N^{2}$ and let $H$ be a finite family of elements of $G$ such that $N^{4} \subset \cup_{z \in H} z N$. By maximality $G=\cup_{j \in J} \cup_{z \in H} y_{j} z N=\cup_{z \in H}\left[\cup_{j \in J} y_{j} N\right] z$. If $f$ is not in $L_{\left(1, p^{\prime}\right)}^{\pi}$, there must be $z_{0}$ in $H$ such that the restriction of $f$ to $\cup_{j \in J} y_{j} N z_{0}$ is not in $L_{\left(1, p_{2}^{\prime}\right)}^{\pi}$, so there must be some subsequence of the $y_{j}$ 's indexed by $Z^{+}$, such that the restriction of $f$ to $\cup_{n=1}^{\infty} y_{n} N z_{0}$ is not in $L_{\left(1, p_{2}^{\prime}\right)}^{\pi}$. Taking $\alpha$ in 4.3 to be $\left\{\left\|f_{y_{n} z_{0} N}\right\|_{1}\right\}$, there is nonnegative $\beta$ in $l_{p_{2}}\left(Z^{+}\right)$such that $\sum_{n=1}^{\infty} \alpha_{n} \beta_{n}=\infty$. Let $g$ be a function defined to be identically $\beta_{n}$ on the set $z_{0}^{-1} y_{n}^{-1} N^{2}$ are zero elsewhere. (Disjointness of $z_{0}^{-1} y_{n}^{-1} N^{2}$ follows from that of $y_{j} N^{2}$.) Clearly $g \in L_{\underline{p}}^{\pi}$ and if $x \in N$ we have

$$
\int_{G}\left|f(y) g\left(y^{-1} x\right)\right| d m(y) \geqslant \sum_{n=1}^{\infty} \int_{y_{n} N z_{0}}|f(y)| g\left(y^{-1} x\right) d m(y) .
$$

If $y \in y_{n} N z_{0}, y^{-1} x \in z_{0}^{-1} y_{n}^{-1} N^{2}$, so $g\left(y^{-1} x\right)=\beta_{n}$. Thus, for all $x$ in $N$,

$$
\int_{G}\left|f(y) g\left(y^{-1} x\right)\right| d m(y)>\sum_{n=1}^{\infty} \alpha_{n} \beta_{n}=\infty \text {. }
$$

Thus $f * g(x)$ is not defined on $N$, a compact set of positive measure; assuming $f$ is not in $L_{\left(1, p_{2}^{\prime}\right)}^{\pi}$ contradicts (a). 
N. Rickert proved [4] that for $1 \leqslant p, q \leqslant \infty, 1 / p+1 / q<1$ on a locally compact group $G$ which is not compact there must be $f$ in $L_{p}(G)$ and $g$ in $L_{q}(G)$ with $f * g$ undefined on a set of positive measure. Theorem 4.4 gives a precise characterization for (IN) groups and show it to be the rule, rather than the exception. For $p, q$ as above, we say $f \in L_{p}(G)$ has a divergent $q$-convolution if there is some $g \in L_{q}(G)$ such that $f * g$ is undefined on a set of positive measure. Immediately from 4.4 we have:

Proposition 4.5. If $1 \leqslant p, q \leqslant \infty, 1 / p+1 / q<1$ and $f \in L_{p}(G)$, $f$ has $a$ divergent $q$-convolution iff $f$ is not in $L_{\left(1, q^{\prime}\right)}^{\pi}(G)$.

Corollary 4.6. The set of $f$ in $L_{p}(G)$ having a divergent $q$-convolution $(1<p$, $q \leqslant \infty, 1 / p+1 / q<1)$ is of second category in $L_{p}(G)$.

Proof. By 4.5 we need only show that $X=L_{p} \cap L_{\left(1, q^{\prime}\right)}^{\pi}$ is one of the first category. With norm $N(f)=\|f\|_{p}+\|f\|_{\left(1, q^{\prime}\right)}^{\pi}, X$ is a Banach space and the natural injection of $X$ into $L_{p}$ is continuous. By 2.11 of [15], since $X$ is not all of $L_{p}$ it must be first category.

5. Product-convolution operators. Let $\Re(G)$ be the locally measurable functions, with locally a.e. equal functions identified. For $h$ in $\mathscr{N}(G), p_{h}$ denotes pointwise multiplication by $h\left(\left(P_{h} g\right)(x)=h(x) g(x)\right)$. For $f$ in $\Re(G), C_{f}$ denotes the operator having as domain the $g$ in $\mathfrak{R}(G)$ such that $f * g$ exists (and is finite) locally a.e. and which maps $g$ to $f * g$. A product-convolution $(P C)$ operator is a composition $P_{h} C_{f}$. We will be concerned with cases where the domain of the $P C$ operator contains, and is restricted to, one of the mixed-norm spaces and the range of this restriction lies in another. In this section we study conditions for the operator to be bounded; in the next, conditions for it to be compact.

THEOREM 5.1. The operator $P_{h} C_{f}$, with $h \in L_{\underline{p}}^{\pi}$ and $f \in L_{\underline{r}}^{\pi}$, bounded from $L_{\underline{q}}^{\pi}$ to $L_{s^{\prime}}{ }^{\prime}$ where $\overline{1} \leqslant \underline{p}, \underline{q}, \underline{r}, \underline{s} \leqslant \bar{\infty}$, if

(a) $1 / p+1 \bar{r}>\overline{1}+1 / \underline{s}-1 / q$ and

(b) $\left(s_{1}, r_{2}\right) \leqslant\left(p_{1}, q_{2}^{\prime}\right)$.

In this case, the bound satisfies

$$
\left\|P_{h} C_{f}\right\|_{\underline{q}, \underline{s}}^{\pi} \leqslant \Gamma(\pi)\|h\|_{\underline{p}}^{\pi}\|f\|_{\underline{\underline{r}}}^{\pi}
$$

$(\Gamma(\pi)$ is as in 4.2).

Proof. (b) implies $1 \leqslant 1 / r_{2}+1 / q_{2}$ so, defining $t$ by

$$
\frac{1}{t_{1}}=\max \left\{0, \frac{1}{r_{1}}+\frac{1}{q_{1}}-1\right\}, \quad \frac{1}{t_{2}}=\frac{1}{r_{2}}+\frac{1}{q_{2}}-1,
$$

(by 4.2) for any $g \in L_{\underline{q}}^{\pi}\|f * g\|_{\underline{t}}^{\pi} \leqslant \Gamma(\pi)\|f\|_{\underline{\underline{L}}}^{\pi}\|g\|_{\underline{q}}^{\pi}$.

(a) and (b) imply $s_{1} \leqslant p_{1}$ and $s_{1} \leqslant t_{1}$. If $s_{1}=\infty$, for any $E$ in $\pi$,

$$
\left\|[h \cdot(f * g)]_{E}\right\|_{s_{1}} \leqslant\left\|h_{E}\right\|_{p_{1}}\left\|(f * g)_{E}\right\|_{t_{1}} .
$$

For $s_{1}<\infty$, since (a) implies $1 / p_{1}+1 / t_{1} \leqslant 1 / s_{1}$ and $|h|_{E}^{s_{1}} \in L_{p_{1} / s_{1}}(E),|f * g|_{E}^{s_{1}} \in$ $L_{t_{1} / s_{1}}(E)$, Hölder's inequality yields 


$$
\begin{aligned}
\left\|\left[|h|^{s_{1}} \cdot|(f * g)|^{s_{1}}\right]_{E}\right\|_{1} & \leqslant\left\||h|_{E}^{s_{1}}\right\|_{p_{1} / s_{1}}\left\||(f * g)|_{E}^{s_{1}}\right\|_{t_{1} / s_{1}} \\
& =\left(\left\|h_{E}\right\|_{p_{1}}\left\|(f * g)_{E}\right\|_{t_{1}}\right)^{s_{1}}
\end{aligned}
$$

so the results hold in this case also. Thus if $s_{2}=\infty$, by the above result,

$$
\begin{aligned}
\|h \cdot(f * g)\|_{\underline{s}}^{\pi} & =\sup _{E \in \pi}\left\|\left[h \cdot(f * g)_{E}\right]\right\|_{s_{1}} \leqslant \sup _{E \in \pi}\left\|h_{E}\right\|_{p_{1}} \sup _{E \in \pi}\left\|(f * g)_{E}\right\|_{t_{1}} \\
& \leqslant\|h\|_{\underline{p}}^{\pi}\|f * g\|_{\underline{\underline{t}}}^{\pi} \leqslant \Gamma(\pi)\|h\|_{\underline{p}}^{\pi}\|f\|_{\underline{\underline{T}}}^{\pi}\|g\|_{\underline{\underline{q}}}^{\pi} .
\end{aligned}
$$

If $s_{2}<\infty$, let $k=\max \left\{1, p_{2} / s_{2}\right\}, n=\max \left\{1, t_{2} / s_{2}\right\}$. Since $1 / p_{2}+1 / t_{2} \geqslant 1 / s_{2}$, by (a) and the definition of $t_{2}, 1 / k+1 / n \geqslant 1$. Again by Hölder's inequality and the preceding result,

$$
\sum_{E \in \pi}\left\|h_{E}\right\|_{p_{1}}^{s_{2}}\left\|(f * g)_{E}\right\|_{t_{1}}^{s_{2}} \leqslant\left[\sum_{E \in \pi}\left\|h_{E}\right\|_{p_{1}}^{s_{2} k}\right]^{1 / k}\left[\sum_{E \in \pi}\left\|(f * g)_{E}\right\|_{t_{1}}^{s_{2} n}\right]^{1 / n},
$$

so

$$
\begin{aligned}
\|h \cdot(f * g)\|_{\underline{s}}^{\pi} & \leqslant\left[\sum_{E \in \pi}\left(\left\|h_{E}\right\|_{p_{1}}\right)^{s_{2}}\left(\left\|(f * g)_{E}\right\|_{t_{1}}\right)^{s_{2}}\right]^{1 / s_{2}} \\
& \leqslant\left[\sum_{E \in \pi}\left(\left\|h_{E}\right\|_{p_{1}}\right)^{s_{2} k}\right]^{1 / s_{2} k}\left[\sum_{E \in \pi}\left(\left\|(f * g)_{E}\right\|_{t_{1}}\right)^{s_{2} n}\right]^{1 / s_{2} n} \\
& \leqslant\left(\sum_{E \in \pi}\left(\left\|h_{E}\right\|_{p_{1}}\right)^{p_{2}}\right)^{1 / p_{2}}\left(\sum_{E \in \pi}\left(\left\|(f * g)_{E}\right\|_{t_{1}}\right)^{t_{2}}\right)^{1 / t_{2}} \\
& =\|h\|_{\underline{p}}^{\pi}\|f * g\|_{\underline{t}}^{\pi} \leqslant \Gamma(\pi)\|h\|_{\underline{p}}^{\pi}\|f\|_{\underline{r}}^{\pi}\|g\|_{\underline{q}}^{\pi} .
\end{aligned}
$$

Corollary 5.2. The operator $P_{h} C_{f}$ with $h \in L_{\underline{p}}^{\pi}$ and $f \in L_{\underline{r}}^{\pi}$ is bounded on $L_{\underline{q}}^{\pi}$, where $\overline{1} \leqslant p, q, \underline{r} \leqslant \bar{\infty}$, provided

(a) $1 / p \overline{+} \overline{1} / \underline{r}>\overline{1}$ and

(b) $\left(q_{1}, r_{2}\right) \leqslant\left(p_{1}, q_{2}\right)$.

In the next section we require a strengthened version of 5.1 which is also of interest in its own right:

THEOREM 5.3. Suppose for $\overline{1} \leqslant p, q, \underline{r}, \underline{s} \leqslant \bar{\infty}$

(a) $1 / \underline{p}+1 / \underline{r}>\overline{1}+1 / \underline{s}-1 \bar{q}$,

(b) $\left(s_{1}, r_{2}\right) \leqslant\left(p_{1}, q_{2}^{\prime}\right)$,

(c) $1 / r_{2}+1 / q_{2} \geqslant 1+1 / s_{2}$,

(d) $\left(s_{1}, r_{2}\right)<\left(p_{1}, \infty\right)$.

Then, for any $f$ in $L_{r}^{\pi}, \varepsilon>0, \theta>0$, there is $\delta>0$ such that for any $h$ in $L_{p}^{\pi}$ for which $\|h\|_{\underline{p}}^{\pi} \leqslant \theta$ and $\|h\|_{(1, \infty)}^{\pi} \leqslant \delta$, then $\left\|P_{h} C_{f}\right\|_{\underline{q}, \underline{s}}^{\pi}<\varepsilon$.

Proof. Since $r_{2}<\infty$ choose $f_{0}$ a bounded function of compact support such that $\left\|f-f_{0}\right\|_{r}^{\pi}<\varepsilon / 2 \theta \Gamma(\pi)$. For $g$ in $L_{q}^{\pi}$ we know by 4.2 that

$$
\left\|f_{0} * g\right\|_{\left(\infty, q_{2}\right)}^{\pi} \leqslant \Gamma(\pi)\left\|f_{0}\right\|_{(\infty, 1)}^{\pi}\|g\|_{\underline{q}}^{\pi}
$$


and

$$
\begin{gathered}
\left\|f_{0} * g\right\|_{\underline{t}}^{\pi} \leqslant \Gamma(\pi)\left\|f_{0}\right\|_{\underline{\underline{L}}}^{\pi}\|g\|_{\underline{q}}^{\pi}, \\
\frac{1}{t_{1}}=\min \left\{0, \frac{1}{r_{1}}+\frac{1}{q_{1}}-1\right\}, \quad \frac{1}{t_{2}}=\frac{1}{r_{2}}+\frac{1}{q_{2}}-1 .
\end{gathered}
$$

We take $\pi$ to be $U-V$ uniform. The usual inequality for $L_{p}$ norms on sets of finite measure yields

$$
\left\|f_{0} * g\right\|_{\left(p_{1} s_{1} /\left(p_{1}-s_{1}\right), t_{2}\right)}^{\pi} \leqslant \Gamma(\pi)\left\|f_{0}\right\|_{\underline{\underline{ }}}^{\pi}\|g\|_{\underline{q}}^{\pi} m(V)^{\left(1 / s_{1}-1 / p_{1}-1 / t_{1}\right)}
$$

since $1 / s_{1}-1 / p_{1} \geqslant 1 / t_{1}$. Let $\alpha=\varepsilon /\left(4 \Gamma(\pi) m(V)^{1 / s_{1}-1 / t_{1}}\left\|f_{0}\right\|_{\underline{L}}^{\pi}\right)$ and choose $\delta>0$ so that

$$
(\delta / \alpha)^{\left(1 / s_{1}-1 / p_{1}\right)} \Gamma(\pi) \theta\left\|f_{0}\right\|_{(\infty, 1)}^{\pi}<\varepsilon / 4 .
$$

For any $h \in L_{\underline{p}}^{\pi}$ such that $\|h\|_{\underline{p}}^{\pi} \leqslant \theta$ and $\|h\|_{(1, \infty)}^{\pi} \leqslant \delta$ and each $E \in \pi$, define $S_{\alpha}=\{x:|h(x)| \geqslant \alpha\}, E_{1}=E \cap S_{\alpha}, E_{2}=E \sim E_{1}$. Then for $i=1,2$

$$
\int_{E_{i}}|h(x)|^{s_{1}}\left|\left(f_{0} * g\right)(x)\right|^{s_{1}} d m(x) \leqslant\left\|\left(h^{s_{1}}\right)_{E_{i}}\right\|_{p_{1} / s_{1}}\left\|\left(f_{0} * g\right)_{E_{i}}^{s_{1}}\right\|_{p_{1} /\left(p_{1}-s_{1}\right)}
$$

so $\left\|\left[h \cdot\left(f_{0} * g\right)\right]_{E_{i}}\right\|_{s_{1}} \leqslant\left\|h_{E_{i}}\right\|_{p_{1}}\left\|\left(f_{0} * g\right)_{E_{i}}\right\|_{p_{1} s_{1} /\left(p_{1}-s_{1}\right)}=\gamma_{i}$. For $i=1$,

$$
\begin{aligned}
\gamma_{1} & \leqslant\left\|h_{E}\right\|_{p_{1}}\left\|\left(f_{0} * g\right)_{E}\right\|_{\infty}\left[m\left(E_{1}\right)\right]^{\left(1 / s_{1}-1 / p_{1}\right)} \\
& \leqslant\left\|h_{E}\right\|_{p_{1}}\left\|\left(f_{0} * g\right)_{E}\right\|_{\infty}\left(\frac{1}{\alpha}\|h\|_{(1, \infty)}^{\pi}\right)^{\left(1 / s_{1}-1 / p_{1}\right)}
\end{aligned}
$$

since $\alpha m\left(E_{1}\right) \leqslant\left\|h_{E}\right\|_{1} \leqslant\|h\|_{(1, \infty)}^{\pi}$. Thus, if $s_{2}<\infty$,

$$
\sum_{E \in \pi}\left(\left\|\left[h\left(f_{0} * g\right)\right]_{E_{1}}\right\|_{s_{1}}\right)^{s_{2}} \leqslant\left(\frac{1}{\alpha}\|h\|_{(1, \infty)}^{\pi}\right)^{\left(s_{2} / s_{1}-s_{2} / p_{1}\right)} \sum_{E \in \pi}\left(\left\|h_{E}\right\|_{p_{1}}\right)^{s_{2}}\left(\left\|\left(f_{0} * g\right)_{E}\right\|_{\infty}\right)^{s_{2}}
$$

But by (1), $\left\{\left\|\left(f_{0} * g\right)_{E}\right\|_{\infty}\right\} \in l_{q_{2}}(\pi)$ and by (a)

$$
\frac{s_{2}}{p_{2}}+\frac{s_{2}}{q_{2}} \geqslant 1+s_{2}\left(1-\frac{1}{r_{2}}\right)=1+\frac{s_{2}}{r_{2}^{\prime}} \geqslant 1
$$

so, using Hölder's inequality,

$$
\begin{aligned}
\sum_{E \in \pi}\left(\left\|h_{E}\right\|_{p_{1}}\right)^{s_{2}}\left(\left\|\left(f_{0} * g\right)_{E}\right\|_{\infty}\right)^{s_{2}} & \leqslant\left(\sum_{E \in \pi}\left(\left\|h_{E}\right\|_{p_{1}}\right)^{p_{2}}\right)^{s_{2} / p_{2}}\left(\sum_{E \in \pi}\left(\left\|\left(f_{0} * g\right)_{E}\right\|_{\infty}\right)^{q_{2}}\right)^{s_{2} / q_{2}} \\
& =\left(\|h\|_{\underline{p}}^{\pi}\right)^{s_{2}}\left(\left\|\left(f_{0} * g\right)\right\|_{\left(\infty, q_{2}\right)}^{\pi}\right)^{s_{2}} \\
& \leqslant\left(\|h\|_{\underline{p}}^{\pi}\right)^{s_{2}}\left(\Gamma(\pi)\left\|f_{0}\right\|_{(\infty, 1)}^{\pi}\|g\|_{\underline{q}}^{\pi}\right)^{s_{2}} \quad(\text { by }(1)) .
\end{aligned}
$$

Thus

$$
\left\|h \cdot\left(f_{0} * g\right)_{S_{\alpha}}\right\|_{\underline{s}} \leqslant\left(\frac{1}{\alpha}\|h\|_{(1, \infty)}^{\pi}\right)^{1 / s_{1}-1 / p_{1}}\|h\|_{\underline{p}}^{\pi} \Gamma(\pi)\left\|f_{0}\right\|_{(\infty, 1)}^{\pi}\|g\|_{\underline{q}}^{\pi}<\frac{\varepsilon}{4}\|g\|_{\underline{q}}^{\pi}
$$

(by (4)). (If $s_{2}=\infty$ the same inequality follows by similar computations.) 
For $i=2, \gamma_{2} \leqslant \alpha(m(V))^{1 / p_{1}}\left\|\left(f_{0} * g\right)_{E}\right\|_{p_{1} s_{1} /\left(p_{1}-s_{1}\right)}$ and if $s_{2}<\infty$ we have

$$
\begin{aligned}
\left\|\left(h \cdot\left(f_{0} * g\right)\right)_{G \sim S_{\alpha}}\right\|_{\underline{s}}^{\pi} & \leqslant \alpha(m(V))^{1 / p_{1}}\left[\sum_{E \in \pi}\left(\left\|\left(f_{0} * g\right)_{E}\right\|_{p_{1} s_{1} /\left(p_{1}-s_{1}\right)}\right)^{s_{2}}\right]^{1 / s_{2}} \\
& \leqslant \alpha(m(V))^{1 / p_{1}}\left\|f_{0} * g\right\|_{\left(p_{1} s_{1} /\left(p_{1}-s_{1}\right), t_{2}\right)}^{\pi} \quad\left(\text { since by }(\mathrm{c}) t_{2} \leqslant s_{2}\right) \\
& \leqslant \alpha(m(V))^{1 / p_{1}} \Gamma(\pi)\left\|f_{0}\right\|_{\underline{\underline{L}}}^{\pi}\|g\|_{\underline{q}}^{\pi} m(V)^{\left(1 / s_{1}-1 / p_{1}-1 / t_{1}\right)} \leqslant \frac{\varepsilon}{4}\|g\|_{\underline{q}}^{\pi} .
\end{aligned}
$$

(Again, the result follows similarly for $s_{2}=\infty$.) Thus in all cases

$$
\left\|h \cdot\left(f_{0} * g\right)\right\|_{\underline{s}}^{\pi} \leqslant\left\|\left(h \cdot\left(f_{0} * g\right)\right)_{S_{\alpha}}\right\|_{\underline{s}}^{\pi}+\left\|\left(h\left(f_{0} * g\right)\right)_{G \sim S_{a}}\right\|_{\underline{s}}^{\pi} \leqslant \frac{\varepsilon}{2}\|g\|_{\underline{q}}^{\pi}
$$

and we have $\left\|P_{h} C_{f_{0}}\right\|_{\underline{q}, \underline{s}}<\varepsilon / 2$.

By 5.1, $\left\|P_{h} C_{\left(f-f_{0}\right)}\right\|_{\underline{q}, \underline{s}}^{\pi} \leqslant \Gamma(\pi)\|h\|_{p}^{\pi}\left\|f-f_{0}\right\|_{r}^{\pi} \leqslant \theta \Gamma(\pi)\left\|f-f_{0}\right\|_{r}^{\pi}<\varepsilon / 2$. Finally, $\left\|P_{h} C_{f}\right\|_{\underline{q}, \underline{s}}^{\pi} \leqslant\left\|P_{h} C_{f_{0}}\right\|_{\underline{q}, \underline{s}}^{\pi}+\left\|P_{h} C_{\left(f-f_{0}\right)}\right\|_{\underline{q}, \underline{s}}^{\bar{\pi}}<\varepsilon$.

Theorem 5.1 tells us that, given the hypotheses on the indices, the bilinear mapping of $L_{p}^{\pi} \times P_{r}^{\pi}$ to the bounded operators from $L_{q}^{\pi}$ to $L_{s}^{\pi}$ defined by $(h, f) \rightarrow P_{h} C_{f}$ is jointly continuous. Theorem 5.3 , however, with slightly stronger hypotheses on the indices, yields only that if the ball $B_{\theta}$ of radius $\theta$ in $L_{p}^{\pi}$ is given the coarser $(1, \infty)$ topology the map from $B_{\theta} \times L_{\underline{r}}^{\pi}$ to these bounded operators is separately continuous. This is precisely what we need for our results on compactness.

We now show that if $P_{h} C_{f}$ is a bounded operator between mixed-norm spaces then $h$ and $f$ must lie in mixed-norm spaces-a result similar to what was proved for $C_{f}$ in 4.4.

Proposition 5.4. Suppose $P_{h} C_{f}$ is a nontrivial bounded operator from $L_{\underline{q}}^{\pi}$ to $L_{\underline{s}}^{\pi}$, with $\overline{1} \leqslant \underline{q}, \underline{s} \leqslant \bar{\infty}$. Then $f$ is in $L_{\left(1, q_{2}^{\prime}\right)}^{\pi}$ and $h$ is in $L_{\left(s_{1}, \infty\right)}^{\pi}$.

Proof. By hypothesis $f * g$ exists locally a.e. for all $g$ in $L_{q}^{\pi}$, so, by $4.4, f$ is in $L_{\left(1, q_{2}^{\prime}\right)}^{\pi}$.

Suppose $s_{1}<\infty$. Choose $g$ in $K(G)$ such that $f * g \neq 0$. Since $f * g$ is continuous there is a $\delta>0$ and a relatively compact open set $W$ such that $|(f(g)(x))| \geqslant \delta$ for $x$ in $W$. Since $h \cdot(f * g)$ is in $L_{s}^{\pi}$, it is locally in $L_{s_{1}}$. Thus if $s_{1}<\infty$

$$
\int_{W}|h(x)|^{s_{1}} d m(x) \leqslant\left(\frac{1}{\delta}\right)^{s_{1}} \int_{W}|h(x)(f * g)(x)|^{s_{1}} d m(x)<\infty .
$$

If $s_{1}=\infty$ a similar inequality holds for essential bounds. By translating $g$ we see that $h$ is locally $L_{s_{1}}$. If there is a sequence $\left\{E_{n}\right\}$ in $\pi$ such that $\left\|h_{E_{n}}\right\|_{s_{1}} \geqslant n$ we can find $\left\{x_{n}\right\}$ such that $\lim _{n \rightarrow \infty}\left\|h_{W x_{n}-1}\right\|_{s_{1}}=\infty$ and calculating as above $\left\|(h \cdot(f * g))_{W x_{n}^{-1}}\right\|_{s_{1}} \rightarrow \infty$ which violate the assumption that $P_{h} C_{f}$ is bounded.

Using 5.1 and 5.4 completely settles one case.

Corollary 5.5. $P_{h} C_{f}$ is bounded from $L_{\infty}$ to $L_{\infty}$ iff $h$ is in $L_{\infty}$ and $f$ is in $L_{1}$.

Proposition 5.4 leads us to examine the necessity of the hypotheses of 5.1 .

We restate the hypotheses:

Let $\overline{1} \leqslant \underline{p}, \underline{q}, \underline{r}, \underline{s} \leqslant \bar{\infty}$ be such that

(1) $1 / p_{1} \overline{+} \overline{1} / r_{1} \leqslant 1+1 / s_{1}-1 / q_{1}$, 
(2) $1 / p_{2}+1 / r_{2} \geqslant 1+1 / s_{2}-1 / q_{2}$,

(3) $s_{1} \leqslant P_{1}$,

(4) $r_{2} \leqslant q_{2}^{\prime}$.

Theorem 5.1 says that if $h$ is in $L_{q}^{\pi}$ and $f$ is in $L_{\underline{r}}^{\pi}, P_{h} C_{f}$ is bounded from $L_{q}^{\pi}$ to $L_{\underline{s}}^{\pi}$. Proposition 5.4 tells us conversely that if $P_{h} C_{f}$ is bounded from $L_{q}^{\pi}$ to $L_{\underline{s}}^{\pi}$ then (3) and (4) hold for some choice of $p$ and $r$ with $h$ in $L_{p}^{\pi}$ and $f$ in $L_{r}{ }^{\pi}$.

We have found examples of "boundary" cases, of functions $h$ and $f$ which come arbitrarily close to satisfying the conditions of (1) and (2) in which $P_{h} C_{f}$ may or may not be bounded.

EXAMPLE 5.6. Let $\left\{\alpha_{n}\right\},\left\{\beta_{n}\right\}$ be sequences such that $-\frac{1}{2}>\alpha_{n}>-1, \lim _{n \rightarrow \infty} \alpha_{n}$ $=-1, \beta_{n}>0$ and $\sum_{n=1}^{\infty} \beta_{n} /\left(1+\alpha_{n}\right)<\infty$. For $0<x \leqslant 1$, define $f(x)=$ $\sum_{n=1}^{\infty} \beta_{n} x^{\alpha_{n}}, h(x)=-\log x$ and let $f(x)=h(x)=0$ elsewhere. Then $h \in L_{p}(R)$ for $1 \leqslant p<\infty$ but $h \notin L_{\infty}(R) ; f \in L_{1}(R)$ but $f \notin L_{q}(R)$ for $1<q \leqslant \infty$. We examine $P_{h} C_{f}$ on $L_{2}(R)$, i.e. $\underline{q}=\underline{s}=\overline{2}$. Then clearly $h$ and $f$ cannot be members of any pair of spaces $L_{p}^{\pi}, L_{r}^{\pi}$ (respectively) for which the conditions (1) and (2) hold, but for any $\varepsilon>0$ there are index pairs $p$ and $\underline{r}$, such that (1) and (2) are violated by less than $\varepsilon$. By choosing particular $g$ in $L_{2}(R)$ it is easy to show the sequence $\left\{\beta_{n}\right\}$ can be chosen so that $P_{h} C_{f}$ is unbounded. The problem is to show that it can be bounded. For this we need:

LEMMA 5.7. Let $g$ be a nonnegative function in $L^{2}(R)$ vanishing on $(0, \infty)$ and let $g^{\wedge}$ denote the increasing rearrangement of $g$ on $(-\infty, 0)$ (i.e. $g^{\wedge}(x)$ is the usual decreasing rearrangement evaluated at $-x)$. Let $f$ be nonnegative, summable, decreasing on $(0, \infty)$ and vanish on $(-\infty, 0)$. Then $(f * g)_{[0, \infty)} \leqslant\left(f * g^{\wedge}\right)_{[0, \infty)}$ and both these functions are decreasing on $(0, \infty)$.

Proof. For $x \geqslant 0$, we have

$$
(f * g)(x)=\int_{0}^{\infty} f(t) g(x-t) d t=\int_{0}^{\infty} f(x+t) g(-t) d t
$$

Since $f$ is decreasing, for $x \geqslant 0$

$$
\int_{0}^{\infty} f(x+t) g(-t) d t \leqslant \int_{0}^{\infty} f(x+t) g^{\wedge}(-t) d t
$$

If $0<x \leqslant y$, then $f(y+t) \geqslant f(x+t) \geqslant 0$ for $t>0$, so $\int_{0}^{\infty} f(y+t) g(-t) d t \geqslant$ $\int_{0}^{\infty} f(x+t) g(-t) d t$ and the functions decrease on $(0, \infty)$.

Suppose $\|g\|_{2}=1$, where $g$ is as in the lemma. Then for $x>0$

$$
1=\int_{0}^{\infty} g^{2}(-t) d t=\int_{0}^{\infty}\left(g^{\wedge}(-t)\right)^{2} d t \geqslant \int_{0}^{\infty}\left(g^{\wedge}(-t)\right)^{2} d t \geqslant x \cdot\left(g^{\wedge}(-x)\right)^{2},
$$

so $g^{\wedge}(-x) \leqslant x^{-1 / 2}$. Thus, by the lemma, for $x>0$

$$
\begin{aligned}
(f * g)(x) & =\int_{0}^{1-x} \sum \beta_{n}(x+t)^{\alpha_{n}} g(-t) d t \\
& \leqslant \int_{0}^{\infty} \sum \beta_{n}(x+t)^{\alpha_{n}} g^{\wedge}(-t) d t \\
& \leqslant \sum \beta_{n} \int_{0}^{\infty}(x+t)^{\alpha_{n}} t^{-1 / 2} d t \\
& =\sum \beta_{n} x^{\alpha_{n}+1 / 2} \int_{0}^{\infty}(1+t)^{\alpha_{n}} t^{-1 / 2} d t .
\end{aligned}
$$


Write $I_{n}=\int_{0}^{\infty}(1+t)^{\alpha_{n}} t^{-1 / 2} d t$. Then, again by the lemma,

$$
\begin{aligned}
\|h \cdot(f * g)\|_{2}^{2} & \leqslant\left\|h \cdot\left(f * g^{\hat{}}\right)\right\|_{2}^{2} \leqslant \int_{0}^{1}\left(\sum \beta_{n} I_{n} x^{\alpha_{n}+1 / 2} \log x\right)^{2} d x \\
& \leqslant \sum \sum \beta_{n} \beta_{m} I_{n} I_{m} \int_{0}^{1} \log ^{2} x x^{\alpha_{n}+\alpha_{m}+1} d x \leqslant \sum \sum \frac{2 \beta_{n} \beta_{m} I_{n} I_{m}}{\left(\alpha_{n}+\alpha_{m}+2\right)^{3}} .
\end{aligned}
$$

Since $-\frac{1}{2}>\alpha_{n}>-1, \alpha_{n} \alpha_{m}<1$ and $\alpha_{n}+\alpha_{m}+2>\left(\alpha_{n}+1\right)\left(\alpha_{m}+1\right)$. Thus

$$
\|h \cdot(f * g)\|_{2}^{2}<2\left(\sum \frac{\beta_{n} I_{n}}{\left(\alpha_{n}+1\right)^{3}}\right)^{2} .
$$

Since $\left\{\beta_{n}\right\}$ was only constrained by $\sum \beta_{n} /\left(1+\alpha_{n}\right)<\infty$, this last sum can be made bounded by appropriate choice of $\left\{\beta_{n}\right\}$ and $P_{h} C_{f}$ is bounded on all positive $g$ in $L^{2}(R)$ with support in $(-\infty, 0)$.

Suppose the support of $g$ is in $(0, \infty)$ and let $\hat{g_{x}}$ be the decreasing rearrangement on $(0, x)$ of the restriction of $g$ to that interval and again assume $\|g\|_{2}=1$. By previous remarks we know $\hat{g_{x}}(t) \leqslant t^{-1 / 2}$ and

$$
\begin{aligned}
(f * g)(x) & \leqslant \int_{0}^{x} f(t) g_{x}^{\wedge}(t) d t \leqslant \int_{0}^{x} f(t) t^{-1 / 2} d t \\
& \leqslant \sum \beta_{n} \int_{0}^{x} t^{\alpha_{n}-1 / 2} d t \leqslant \sum \beta_{n} \frac{x^{\alpha_{n}+1 / 2}}{\left(\alpha_{n}+\frac{1}{2}\right)} .
\end{aligned}
$$

Much as before

$$
\begin{aligned}
\|h \cdot(f * g)\|_{2}^{2} & \leqslant \sum \sum \frac{\beta_{n} \beta_{m}}{\left(\alpha_{n}+\frac{1}{2}\right)\left(\alpha_{m}+\frac{1}{2}\right)} \int_{0}^{1} x^{\alpha_{n}+\alpha_{m}+1} \log ^{2} x d x \\
& \leqslant \sum \sum \frac{2 \beta_{n} \beta_{m}}{\left(\alpha_{n}+\frac{1}{2}\right)\left(\alpha_{m}+\frac{1}{2}\right)\left(\alpha_{n}+\alpha_{m}+2\right)^{3}} \leqslant 2\left(\sum \frac{\beta_{n}}{\left(\alpha_{n}+\frac{1}{2}\right)\left(\alpha_{n}+1\right)^{3}}\right)^{2} .
\end{aligned}
$$

Again, $\left\{\beta_{n}\right\}$ can be chosen so this sum is bounded, as well as the previous ones. Since any $g$ in $L^{2}(R)$ can be constructed as linear combinations of positive functions having supports in the two half-lines, for $\left\{\beta_{n}\right\}$ appropriately chosen we have $P_{h} C_{f}$ bounded from $L^{2}$ to $L^{2}$.

6. Compactness of $P C$ operators. In this section we give necessary and sufficient conditions for compactness of $P C$ operators which satisfy the sufficient conditions for boundedness given in 5.1. We thus consider $P_{h} C_{f}: L_{\underline{q}}^{\pi} \rightarrow L_{\underline{s}}{ }^{\pi}$ with $h$ in $L_{\underline{p}}{ }^{\pi}$ and $f$ in $L_{\underline{r}}{ }^{\pi}$ where the indices satisfy

(1) $1 / p_{1}+1 / r_{1} \leqslant 1+1 / s_{1}-1 / q_{1}$,

(2) $1 / p_{2}+1 / r_{2} \geqslant 1+1 / s_{2}-1 / q_{2}$,

(3) $s_{1} \leqslant p_{1}$,

(4) $r_{2} \leqslant q_{2}^{\prime}$.

Because we do not know enough about compact sets in $L_{\infty}$ we sometimes further restrict attention to cases which also satisfy

(5) $s_{1}<\infty$ and

(6) $s_{2}<\infty$. 
If the "global" indices $p_{2}$ and $r_{2}$ are finite, the operators are always compact. Except for the possibility that one or both of "local" indices $p_{1}$ and $r_{1}$ may be infinite, the proof is similar to classical results for kernel operators. We include the proof because of this complication.

Definition 6.1. We say a measurable function $F$ on $G \times G$ is in $L_{u, v}^{\pi}(G \times G)$ if $F(x, \cdot)$ is in $L_{\underline{u}}^{\pi}$ for almost all $x$ and the function $x \rightarrow\|F(x, \cdot)\|_{u}^{\pi}$ is in $L_{v}^{\pi}$. The $L_{v}^{\pi}$ norm of this latter function will be denoted $\|F\|_{(\underline{u}, v)}^{\pi}$.

LemMA 6.2. For $\overline{1} \leqslant s, \underline{q} \leqslant \bar{\infty}$ and $F$ in $L_{q^{\prime}, s}^{\pi}(G \times G)$, the integral operator $T$ : $L_{\underline{q}}^{\pi} \rightarrow L_{\underline{s}}^{\pi}$ defined by $\operatorname{Tg}(x)=\int_{G} F(x, y) g(y)$ dy is bounded and $\|T\| \leqslant\|F\|_{\left(q^{\prime}, s\right)}^{\pi}$.

Proof. By 3.7(b) and the hypotheses on $F,|T g(x)| \leqslant\|F(x,-)\|_{\underline{q}^{\prime}}^{\pi}\|g\|_{\underline{q}}^{\pi}$ a.e. on $G$, so $\|T g\|_{\underline{s}}^{\pi} \leqslant\|g\|_{q}^{\pi}\|F\|_{\left(q^{\prime}, s\right.}^{\pi}$.

REMARK 6.3. If $F$ is continuous with compact support, then the transformation defined in the lemma is compact.

Proof. $F$ can be approximated uniformly by "degenerate" kernels composed from functions in $K(G)$. The norm bound of the lemma then shows that $T$ is approximated in norm by the corresponding operators of finite rank.

THEOREM 6.4. Under conditions (1) through (5) above, if $p_{2}<\infty, r_{2}<\infty$ then $P_{h} C_{f}$ is compact.

Proof. Suppose first that $r_{1}<\infty$. By 3.7(e) choose, for any $\varepsilon>0, f_{e}$ in $K(G)$ such that $\left\|f-f_{\varepsilon}\right\|_{r}^{\pi} \leqslant \varepsilon /\left(2\|h\|_{p}^{\pi} \Gamma(\pi)\right)$. Choose a finite $\hat{p_{1}} \leqslant p_{1}$ such that $s_{1} \leqslant p_{1}$; $1 / p_{1} \leqslant 1+1 / s_{1}-1 / q_{1}$. Again by 3.7(e) we can choose $h_{\varepsilon}$ in $K(G)$ such that $\left\|h-h_{\varepsilon}\right\|_{\left(p_{1}, p_{2}\right)}^{\pi}<\varepsilon / 2 \Gamma(\pi)\left\|f_{\varepsilon}\right\|_{\left(\infty, r_{2}\right)}^{\pi}$. Since $f_{\varepsilon}$ is in $L_{\left(\infty, r_{2}\right)}^{\pi},\left(h-h_{\varepsilon}\right)$ is in $L_{\left(p_{1}, p_{2}\right)}^{\pi}$, and $p_{1}$ was chosen so that $\left(\hat{p_{1}}, p_{2}\right), \underline{q},\left(\infty, r_{2}\right), \underline{s}$ satisfy the conditions of 5.1 , we have

$$
\left\|P_{h} C_{f_{e}}-P_{h_{\varepsilon}} C_{f_{e}}\right\| \leqslant \Gamma(\pi)\left\|h-h_{\varepsilon}\right\|_{\left(p_{1}, p_{2}\right)}^{\pi}\|f\|_{\left(\infty, r_{2}\right)}^{\pi}<\varepsilon / 2
$$

But again, using 5.1,

$$
\left\|P_{h} C_{f}-P_{h} C_{f_{e}}\right\| \leqslant \Gamma(\pi)\|h\|_{\underline{p}}^{\pi}\left\|f-f_{e}\right\|_{\underline{r}}^{\pi}<\varepsilon / 2,
$$

so $P_{h} C_{f}$ can be approximated in norm by the compact $P_{h_{e}} C_{f_{e}}$ and is itself compact.

Now suppose $r_{1}=\infty$. If also $p_{1}=\infty$, (1) becomes a strict inequality, we can find $\hat{p_{1}}<\infty$ such that $\left(\hat{p_{1}}, p_{2}\right), \underline{r}, \underline{q}, \underline{s}$ satisfy the conditions of the theorem and $h$ is also in $L_{\left(p_{1}, p_{2}\right)}^{\pi}$. Thus we may suppose without loss of generality that $p_{1}<\infty$. Given $\varepsilon>0$, we can find $h_{\varepsilon}$ in $K(G)$ such that $\left\|h-h_{\varepsilon}\right\|_{p}^{\pi}<\varepsilon / 2 \Gamma(\pi)\|f\|_{\underline{r}}^{\pi}$. Since $p_{1}<\infty$ we can find $r_{1}<\infty$ such that $1 / r_{1} \leqslant 1+1 / s_{1}-1 / q_{1}$. Choose $f_{\varepsilon}$ in $K(G)$ such that $\left\|f-f_{\varepsilon}\right\|_{\left(r_{1}, r_{2}\right)}^{\pi}<\varepsilon /\left(2 \Gamma(\pi)\left\|h_{\varepsilon}\right\|_{\left(\infty, p_{2}\right)}^{\pi}\right)$. Since $\left(f-f_{\varepsilon}\right)$ is in $L_{\left(r_{1}, r_{2}\right)}^{\pi}, h_{\varepsilon}$ is in $L_{\left(\infty, p_{2}\right)}^{\pi}$ and $\left(\infty, p_{2}\right), q,\left(\hat{r}_{1}, r_{2}\right), s$ satisfy the hypotheses of 5.1 we have $\left\|P_{h_{k}} C_{f}-P_{h_{k}} C_{f_{e}}\right\|<$ $\Gamma(\pi)\left\|h_{\varepsilon}\right\|_{\left(\infty, p_{2}\right)}^{\bar{\pi}}\left\|f-f_{\varepsilon}\right\|_{\left(r_{1}, r_{2}\right)}^{\pi} \leqslant \varepsilon / 2$. Moreover $\left\|P_{h} C_{f}-P_{h_{\varepsilon}} C_{f}\right\| \leqslant \Gamma(\pi)\left\|h-h_{e}\right\|_{\underline{p}}^{\pi}\|f\|_{\underline{r}}^{\pi}$ $<\varepsilon / 2$ and, as before, $P_{h} C_{f}$ is compact.

More interesting results are obtained when either $p_{2}$ or $r_{2}$ is infinite. We now consider these cases when conditions (1) through (6) are met. 
LEMMA 6.5. If $s_{1}, s_{2}<\infty$ and $\alpha, \beta$ are members of $L_{s}{ }^{\pi}$ having disjoint (essential) support, then

$$
\|\alpha\|_{\underline{s}}^{\pi}+\|\beta\|_{\underline{s}}^{\pi} \geqslant\|\alpha \pm \beta\|_{\underline{s}}^{\pi} \geqslant 2^{\left(1 / s_{1}+1 / s_{2}-2\right)}\left[\|\alpha\|_{\underline{s}}^{\pi}+\|\beta\|_{\underline{s}}^{\pi}\right]
$$

Proof. The first inequality is clear. For the second, we know by 2.1

$$
\begin{aligned}
\|\alpha \pm \beta\|_{\underline{s}}^{\pi} & =\left[\sum_{E \in \pi}\left(\int_{E}|\alpha(x) \pm \beta(x)|^{s_{1}} d m(x)\right)^{s_{2} / s_{1}}\right]^{1 / s_{2}} \\
& =\left[\sum_{E \in \pi}\left(\left(\left\|\alpha_{E}\right\|_{s_{1}}\right)^{s_{1}}+\left(\left\|\beta_{E}\right\|_{s_{1}}\right)^{s_{1}}\right)^{s_{2} / s_{1}}\right]^{1 / s_{2}} \\
& \geqslant\left[\sum_{E \in \pi}\left[2^{\left(1 / s_{1}-1\right)}\left\|\alpha_{E}\right\|_{s_{1}}+\left\|\beta_{E}\right\|_{s_{1}}\right]^{s_{2}}\right]^{1 / s_{2}} \\
& \geqslant 2^{\left(1 / s_{1}-1\right)}\left[\sum_{E \in \pi}\left(\left\|\alpha_{E}\right\|_{s_{1}}\right)^{s_{2}}+\left(\left\|\beta_{E}\right\|_{s_{1}}\right)^{s_{2}}\right]^{1 / s_{2}} \\
& \geqslant 2^{\left(1 / s_{1}+1 / s_{2}-2\right)}\left[\left(\sum_{E \in \pi}\left(\left\|\alpha_{E}\right\|_{s_{1}}\right)^{s_{2}}\right)^{1 / s_{2}}+\left(\sum_{E \in \pi}\left(\left\|\beta_{E}\right\|_{s_{1}}\right)^{s_{2}}\right)^{1 / s_{2}}\right] \\
& =2^{\left(1 / s_{1}+1 / s_{2}-2\right)}\left[\|\alpha\|_{\underline{s}}^{\pi}+\|\beta\|_{\underline{s}}^{\pi}\right] .
\end{aligned}
$$

Since $G$ is an (IN) group, the operator $S$ defined on locally measurable functions by $(S f)(x)=f^{\sim}(x)=f\left(x^{-1}\right)$ leaves all the spaces $L_{\underline{p}}{ }^{\pi}(G)$ invariant and is bounded on each. Then $\left(S C_{f} S g\right)(x)=\int_{G} f(y) g(x y) d m(y)$ and $S P_{h} S=P_{h^{-}}$so $S P_{h} C_{f} S=$ $P_{h} S C_{f} S$. These will be used in the next proposition. We will also need $Q(t, a, f)$ defined for all $t$ and $a$ in $G$ and $f$ in $L_{\underline{r}}^{\pi}$, by a fixed $h$ in $L_{\underline{p}}^{\pi}$ and a fixed $g$ of compact essential support in $L_{\underline{q}}^{\pi}$ by

$$
\begin{aligned}
Q(t, a, f)= & \left\|P_{h} S C_{f} S\left({ }_{a} g-{ }_{t} g\right)\right\|_{\underline{s}}^{\pi} \\
& -2^{\left(1 / s_{1}+1 / s_{2}-2\right)}\left[\left\|P_{h} S C_{f} S\left({ }_{a} g\right)\right\|_{\underline{s}}^{\pi}+\left\|P_{h} S C_{f} S\left({ }_{l} g\right)\right\|_{\underline{s}}^{\pi}\right]
\end{aligned}
$$

where (1) through (6) all hold, and $r_{2}<\infty$.

LEMMA 6.6. $\underline{\lim }_{t \rightarrow \infty} Q(t, a, f) \geqslant 0$.

Proof. Since $r_{2}<\infty$, there is a compact set $L$ for any $\delta>0$ such that $\left\|f-f_{L}\right\|_{r}^{\pi}<\delta$. Then

$$
\begin{aligned}
\mid Q(t, a, f)- & Q\left(t, a, f_{L}\right) \mid \\
& \leqslant\left(\left\|P_{h} S C_{\left(f-f_{L}\right)} S\left({ }_{a} g\right)\right\|_{\underline{s}}^{\pi}+\left\|P_{h} S C_{\left(f-f_{L}\right)} S\left({ }_{t} g\right)\right\|_{\underline{s}}^{\pi}\right)\left(1+2^{1 / s_{1}+1 / s_{2}-2}\right) \\
& \leqslant\left\|P_{h^{\top}}\right\|\|S\|\left\|S^{-1}\right\|\left(\left\|_{a} g\right\|_{\underline{q}}^{\pi}+\|t g\|_{\underline{q}}^{\pi}\right)\left(1+2^{1 / s_{1}+1 / s_{2}-2}\right)\left\|f-f_{L}\right\|_{\underline{\underline{L}}}^{\pi} \\
& \leqslant\|h\|_{\underline{p}}^{\pi}\|S\|^{2} \lambda\|g\|_{\underline{q}}^{\pi}\left(1+2^{1 / s_{1}+1 / s_{2}-2}\right)\left\|f-f_{L}\right\|_{r}^{\pi} \\
& <\delta\|h\|_{\underline{\underline{r}}}^{\pi}\|S\|^{2}\|g\|_{\underline{q}}^{\pi}\left(1+2^{1 / s_{1}+1 / s_{2}-1}\right) .
\end{aligned}
$$


By 3.14, $\lambda>0$ can be chosen independent of $a$ and $t$, so by choice of $\delta$ this last quantity is less than $\varepsilon$. The essential support of $S C_{f_{L}} S\left({ }_{a} g\right)$ is contained in $a^{-1} K L^{-1}$ and that of $S C_{f_{L}} S\left({ }_{t} g\right)$ in $t^{-1} K L^{-1}$. Take $K_{e}$ to be $K L^{-1} L K^{-1} a$; then if $t$ is not in $K_{e}$, $P_{h} S C_{f_{L}} S\left({ }_{a} g\right)$ and $P_{h} S C_{f_{L}} S\left({ }_{t} g\right)$ are in $L_{\underline{s}}{ }^{\pi}$ and have disjoint essential supports. Thus by 6.5, $Q\left(t, a, f_{L}\right) \geqslant 0$. Hence if $t \notin K_{2}, Q(t, a, f) \geqslant-\varepsilon$.

Proposition 6.7. If (1) through (6) hold and $p_{2}=\infty, f \in L_{r}^{\pi}, f \neq 0, h \in L_{p}^{\pi}$, then $P_{h} C_{f}$ from $L_{q}^{\pi}$ to $L_{\underline{s}}^{\pi}$ is not compact if $h \notin L_{\left(s_{1}, 0\right)}^{\pi}$.

Proof. By discussion preceding 6.6 and the invariance of $L_{\left(s_{1}, 0\right)}^{\pi}$ under $S$, it suffices to show $P_{h} S C_{f} S$ is not compact.

Choose $g \neq 0$ in $K(G)$ with support $L$. Then $S C_{f} S g$ is continuous, so there is an open $W$ and $\varepsilon>0$ such that $\left|S C_{f} S g\right| \geqslant \varepsilon$ on $W$. There is a symmetric relatively compact neighborhood $U$ of the identity and $x_{0}$ in $W$ such that $x_{0} U^{4} \subset W$. Let $\pi$ and $F$ be as in 3.4 with $V=U^{2}$.

If $x_{0} \in E^{\prime} \in \pi, x_{E^{\prime}} \in x_{0} U^{2}$, so $E^{\prime} \subset x_{0} U^{4} \subset W$. Thus

$$
\left\|P_{h} S C_{f} S g\right\|_{\underline{s}}^{\pi} \geqslant\left[\int_{E^{\prime}}|h(x)|^{s_{1}}\left|\left(S C_{f} S\right)(g)\right|^{s_{1}} d m(x)\right]^{1 / s_{1}} \geqslant \varepsilon\left\|h_{E^{\prime}}\right\|_{s_{1}} .
$$

If we write $z(E)=x_{E}, x_{E}^{-1}$, we have similarly

$$
\left\|P_{h} S C_{f} S\left(_{z(E)} g\right)\right\|_{\underline{s}}^{\pi} \geqslant\left[\int_{E}|h(x)|^{s_{1}}\left|\left(S C_{f} S\left({ }_{z(E)} g\right)\right)(x)\right|^{s_{1}} d m(x)\right]^{1 / s_{1}} \geqslant \varepsilon\left\|h_{E}\right\|_{s_{1}}
$$

for if $x \in E,\left(S C_{f} S(z(E) g)\right)(x)=\left(S C_{f} S g\right)\left(x_{E^{\prime}} x_{E}^{-1} x\right)$ and $x_{E^{\prime}} x_{E}^{-1} x \in x_{E^{\prime}} U^{2} \subset W$. Note that:

(i) If $D$ is a compact set, there is a finite subset, $J(D)$, of $\pi$ such that if $z(E) \in D$ then $E \in J(D)$.

(ii) Since $h \notin L_{\left(s_{1}, 0\right)}^{\pi}$, there is $\delta>0$ such that for any finite subset of $\pi$, there is a member $E$ of $\pi$ not in that subset such that $\left\|h_{E}\right\|_{s_{1}}>\delta$.

Let $c=\varepsilon \delta 2^{1 / s_{1}+1 / s_{2}-2}$ and choose $E_{1}$ in $\pi$ such that $\left\|h_{E_{1}}\right\|_{s_{1}}>\delta$. Let $z_{1}=z\left(E_{1}\right)$ and (by 6.6) choose a compact $\Gamma$ such that $Q(t, z, f) \geqslant-c$ for $t$ outside $\Gamma$. By (i) and (ii) we can pick $E_{2} \notin J(\Gamma)$ with $\left\|h_{E_{2}}\right\|_{s_{1}} \geqslant \delta$. Letting $z_{2}=z\left(E_{2}\right)$ and using computations made above we see that $\left\|P_{h} S C_{f} S\left({ }_{z_{i}} g\right)\right\|_{s}^{\pi} \geqslant \varepsilon \delta$ for $i=1,2$, and $Q\left(z_{2}, z_{1}, f\right) \geqslant-c$ since $z_{2}$ is not in $\Gamma$. But by definition of $Q$ we have

$$
\left\|P_{h} S C_{f} S\left(z_{1} g\right)-P_{h} S C_{f} S\left({ }_{z_{2}} g\right)\right\|_{s}^{\pi} \geqslant 2^{1 / s_{1}+1 / s_{2}-2}(2 \varepsilon \delta)-c=c .
$$

Suppose we have chosen $E_{1}, E_{2}, \ldots, E_{n-1}$ in $\pi$ such that, writing $z_{i}$ for $z\left(E_{i}\right)$ we have, for $i \neq j$, (a) $\left\|P_{h} S C_{f} S\left(z_{i} g\right)\right\|_{\underline{s}}^{\pi} \geqslant \varepsilon \delta$ and (b) $\left\|P_{h} S C_{f}\left(z_{i} g\right)-P_{h} S C_{f} S\left(z_{z_{j}} g\right)\right\|_{s}^{\pi}>c$ where $i$ and $j$ run over $1,2, \ldots, n-1$. By 6.6, choose $\Gamma$ compact such that $Q\left(t, z_{i}, f\right) \geqslant-c$ for $t$ outside $\Gamma$ and for $i=1,2, \ldots, n-1$. By (i) and (ii), choose $E_{n} \notin J(\Gamma)$ such that $\left\|h_{E_{n}}\right\|_{s_{1}} \geqslant \delta$. As above (a) and (b) then hold for $E_{1}, \ldots, E_{n}$. We have, by induction, a bounded sequence $\left\{z_{n} g\right\}$ in $L_{q}^{\pi}$ such that $\left\{P_{h} S C_{f} S\left(z_{n} g\right)\right\}$ has no convergent subsequence, so $P_{h} S C_{f} S$ is not compact.

We now prove the main result of this section. We give two equivalent versions. 
TheOREM A. Let $p, q, \underline{r}, \underline{s}$, satisfy conditions (1) through (6) and $p_{2}=+\infty$. For $f$ in $L_{r}^{\pi}, h$ in $L_{p}^{\pi}$.

(a) If $s_{1}<p_{1}, P_{h} C_{f}$ is compact from $L_{q}^{\pi}$ to $L_{\underline{s}}^{\pi}$ iff $h$ is in $L_{(1,0)}^{\pi}$.

(b) If $s_{1}=p_{1}, P_{h} C_{f}$ is compact from $L_{q}^{\pi}$ to $L_{\underline{s}}^{\pi}$ iff $h$ is in $L_{\left(p_{1}, 0\right)}^{\pi}$.

THEOREM $\mathrm{A}^{\prime}$. Under the above conditions, $P_{h} C_{f}$ is compact from $L_{q}^{\pi}$ to $L_{\underline{s}}^{\pi}$ iff $h$ is in $L_{\left(s_{1}, 0\right)}^{\pi}$.

Proof of A. (a) Suppose $h$ is in $L_{(1,0)}^{\pi}$. Then there is a sequence $K_{n}$ of compact sets such that $\left\|h-h_{n}\right\|_{(1, \infty)}^{\pi}<1 / n$ where $h_{n}$ is the restriction of $h$ to $K_{n}$. The hypotheses of 5.3 hold, so $\left\|P_{h} C_{f}-P_{h_{n}} C_{f}\right\|=\left\|P_{\left(h-h_{n}\right)} C_{f}\right\| \rightarrow 0$. On the other hand, $P_{h_{n}} C_{f}$ is compact by 6.4 so $P_{h} C_{f}$ is also compact.

Conversely, if $h$ is not in $L_{(1,0)}^{\pi}$, it is not in $L_{\left(s_{1}, 0\right)}^{\pi}$ so $P_{h} C_{f}$ is not compact by 4.7 .

(b) If $h$ is in $L_{\left(p_{1}, 0\right)}^{\pi}$, we can find $h_{n}$ as in (a) such that $\left\|h-h_{n}\right\|_{p}^{\pi} \rightarrow 0$. By 5.1, $\left\|P_{h} C_{f}-P_{h_{n}} C_{f}\right\| \leqslant \Gamma(\pi)\|f\|_{r}^{\pi}\left\|h-h_{n}\right\|_{p}^{\pi} \rightarrow 0$. Since $P_{h_{n}} C_{f}$ is compact by 6.4, $P_{h} C_{f}$ is also. The converse is 6.7.

Proof of $\mathrm{A}^{\prime}$. The direct part is 6.7. For the converse, let $s_{1}=p_{1}$. By (b) of Theorem A, $P_{h} C_{f}$ is compact. For $s_{1}<p_{1}, h$ in $L_{\left(s_{1}, 0\right)}^{\pi}$ implies $h$ in $L_{(1,0)}^{\pi}$ so, by (a) of Theorem $\mathrm{A}, P_{h} C_{f}$ is compact.

The case remaining are those in which $r_{2}=+\infty$ which implies $q_{2}=1$. We solve the compactness problem for all such cases when $G$ is first countable.

Definition 6.8. Let $X$ be a translation invariant topological vector space of measurable functions. A member of $X$ will be called translation-continuous in $X$ provided its orbits under both left and right translations define continuous maps of $G$ to $X$.

By 3.13 every member of $L_{p}^{\pi}$ is translation-continuous if $\overline{1} \leqslant p<\bar{p}$. Some members of $L_{\infty}(G)$ are not. We characterize the situation for the case $p_{2}=\infty$. (We only sketch the proof.)

Lemma 6.9. Let $\pi$ and $F$ be as in 3.4. For $f$ in $L_{(p, \infty)}^{\pi}, 1 \leqslant p<\infty$, the following are equivalent:

(a) $f$ is translation-continuous in $L_{(p, \infty)}^{\pi}$.

(b) $f$ is the limit in $L_{(p, \infty)}^{\pi}$ of uniformly continuous functions on $G$.

(c) $\left\{{ }_{x_{E}^{-}} f_{E}: E \in \pi\right\}$ is a compact set in $L_{p}(V)$.

Proof. Clearly uniformly continuous functions are translation-continuous and it follows easily that norm limits in $L_{(p, \infty)}^{\pi}$ also have the property. Let $\left\{G_{\alpha}: \alpha \in A\right\}$ be a net of neighborhoods of the identity with $\cap_{\alpha \in A} G_{\alpha}=\{e\}$ and let $g_{\alpha}$ be the characteristic function of $G_{\alpha}$, normalized in $L_{1}$. A modification of the classical argument for $L_{1}$ shows $g_{\alpha} * f$ converges to $f$ in $L_{(p, \infty)}^{\pi}$ and clearly $g_{\alpha} * f$ are uniformly continuous. Thus (a) and (b) are equivalent.

Note that $f$ in $L_{(p, \infty)}^{\pi}$ means precisely that the set $\left\{x_{x^{-1}} f_{E}\right\}$ is bounded in $L_{p}(V)$ and thus satisfies, as a subset of $L_{p}(G)$, conditions (a) and (c) of 3.13. It can be shown (we omit the details) that the set satisfies condition (b) of 3.13 iff $f$ is translation-continuous. Thus the set is compact iff $f$ is translation-continuous and (a) is equivalent to (c). 
TheOREM B. Let $p, q, \underline{r}, \underline{s}$ satisfy conditions (1) through (6), $r_{2}=+\infty$. With $f$ in $L_{r}^{\pi}, h \neq 0$ in $L_{p}^{\pi}$, for the operator $P_{h} C_{f}$ to be compact from $L_{q}^{\pi}$ to $L_{s}^{\pi}$ it is sufficient and, if $G$ is first countable, it is necessary that $f$ be translation-continuous in the space of bounded local multipliers $L M_{q_{1}, s_{1} ; \infty}^{\pi}$ (cf. Definition 3.19).

Proof. By the hypotheses, we have $q_{2}=1, p_{2} \leqslant s_{2}<\infty$. Also $1 / r_{1}+1 / q_{1}-$ $1 / s_{1} \leqslant 1-1 / p_{1} \leqslant 1$. Since $f$ is locally $r_{1}$ it follows from Young's inequality that if $E$ and $F$ are relatively compact sets of positive measure, $\Lambda_{F} C_{F}$ maps $L_{q_{1}}(E)$ into $L_{s_{1}}(F)$ and its norm is bounded by $\left\|f_{F E^{-1}}\right\|_{r_{1}}$. For any $H$ in $\pi$,

$$
\begin{aligned}
\|f\|_{q_{1}, s_{1} ; \infty}^{\pi, H} & =\sup _{E \in \pi}\left\|\Lambda_{H} C_{f} \Lambda_{E}\right\|_{q_{1}, s_{1}} \leqslant \sup _{E \in \pi}\left\|f_{H E^{-1}}\right\|_{r_{1}} \\
& \leqslant \sup _{E \in \pi} \sum_{E^{\prime} \in S(E, H)}\left\|f_{E^{\prime}}\right\|_{r_{1}} \leqslant \Gamma(\pi)\|f\|_{\underline{r}}^{\pi}<\infty
\end{aligned}
$$

(where $S(E, H)$ and $\Gamma(\pi)$ are as in 4.1). Thus $f$ is in $L M_{q_{1}, s_{1} ; \infty}^{\pi}$.

Suppose $f$ is translation-continuous. Assume first $p_{1}<\infty$ and let $g$ be in the unit ball of $L_{q}^{\pi}$. For any $x$ in $G$, we have

$$
\begin{aligned}
\left\|_{x}\left(P_{h} C_{f} g\right)-P_{h} C_{f} g\right\|_{\underline{s}}^{\pi} & =\left\|\left({ }_{x} h\right) \cdot\left({ }_{x} f * g\right)-h \cdot(f * g)\right\|_{s}^{\pi} \\
& \leqslant\left\|\left({ }_{x} h-h\right) \cdot\left({ }_{x} f * g\right)\right\|_{\underline{s}}^{\pi}+\left\|h \cdot\left[\left({ }_{x} f-f\right) * g\right]\right\|_{s}^{\pi} \\
& \leqslant \Gamma(\pi)\left\|_{x} h-h\right\|_{\underline{\underline{s}}}^{\pi}\left\|_{x} f\right\|_{\underline{\underline{s}}}^{\pi}+\|h\|_{\underline{\underline{p}}}^{\pi}\left\|\left({ }_{x} f-f\right) * g\right\|_{\underline{\underline{t}}}^{\pi}
\end{aligned}
$$

(as in the proof of 5.1) where $1 / t_{1}=\max \left(0,1 / r_{1}+1 / q_{1}-1\right)$ and $1 / t_{2}=1 / r_{2}+$ $1 / q_{2}-1$. By the hypotheses of the theorem, however, we have in this case $t_{1} \geqslant s_{1}$ and $t_{2}=\infty$, so

$$
\left\|{ }_{x}\left(P_{h} C_{f} g\right)-P_{h} C_{f} g\right\|_{\underline{s}}^{\pi} \leqslant \Gamma(\pi)\left\|_{x} h-h\right\|_{\underline{p}}^{\pi}\left\|_{x} f\right\|_{r}^{\pi}+\|h\|_{\underline{p}}^{\pi}\left\|\left({ }_{x} f-f\right) * g\right\|_{\left(s_{1}, \infty\right)}^{\pi},
$$

and we know

$$
\begin{aligned}
\left\|\left({ }_{x} f-f\right) * g\right\|_{\left(s_{1}, \infty\right)}^{\pi} & =\sup _{E \in \pi} \sum_{E^{\prime} \in \pi}\left\|\left(\left({ }_{x} f-f\right) * g_{E^{\prime}}\right)_{E}\right\|_{s_{1}} \\
& \leqslant \sup _{E \in \pi} \sum_{E^{\prime} \in \pi}\left\|\Lambda_{E} C_{\left(x_{x} f-f\right)} \Lambda_{E^{\prime}}\right\|_{q_{1}, s_{1}}\left\|g_{E^{\prime}}\right\|_{q_{1}} .
\end{aligned}
$$

Let $N$ be the compact invariant neighborhood for the (IN) group $G$. We know that $N \subseteq U y_{1} \cup U y_{2} \cup \ldots \cup U y_{k}$ and $V \subseteq z_{1} N \cup z_{2} N \cup \ldots \cup z_{r} N$ for some finite sets of $y$ 's and $z$ 's. Then given any $E_{0}$ in $\pi$ any other member $E$ is contained in the set $\cup_{i=1}^{r} \cup_{j=1}^{k} E_{0} y_{j} x_{E_{0}}^{-1} x_{E} z_{i}$ consisting of $r k$ right translates of $E_{0}$ (where, as usual, we have $\pi$ and the set $F$ of $x_{E}$ as in 3.4). Let $S\left(E^{\prime}, z\right)=\left\{E^{\prime \prime} \in \pi\right.$ : $\left.E^{\prime} z \cap E^{\prime \prime} \neq \varnothing\right\}$. By 3.4 , the cardinality of $S\left(E^{\prime}, z\right)$ is bounded by $r n_{F}(N, V)$, since $E^{\prime \prime} \in S\left(E^{\prime}, z\right)=\cup_{i=1}^{r}\left(x_{E^{\prime}} z_{i} N \cap E^{\prime \prime}\right) \neq \varnothing$, and the bound is independent of $z$ and $E^{\prime}$. Thus for some choice of $k r$ elements $\left\{g_{j}\right\}$ in $G$, depending on $E$, we have 


$$
\begin{aligned}
\left\|\Lambda_{E} C_{(x f-f)} \Lambda_{E^{\prime}}\right\|_{q_{1}, s_{1}} & \leqslant \sum_{j=1}^{k r}\left\|\Lambda_{E_{0} g_{j}} C_{(x f-f)} \Lambda_{E^{\prime}}\right\|_{q_{1}, s_{1}} \\
& =\sum_{j=1}^{k r}\left\|\Lambda_{E_{0}} C_{\left(x_{x} f-f\right)} \Lambda_{E^{\prime} g_{j}^{-1}}\right\|_{q_{1}, s_{1}} \leqslant \sum_{j=1}^{k r} \sum_{E^{\prime \prime} \in S\left(E^{\prime}, g_{j}^{-1}\right)}\left\|\Lambda_{E_{0}} C_{\left(x_{x} f-f\right)}\right\|_{E^{\prime \prime}} \\
& \leqslant k r^{2} n_{F}(N, V)\left\|_{x} f-f\right\|_{q_{1}, s_{1} ; \infty}^{\pi, E_{0}} \quad \text { (by } 3.15 \text { and 3.16). }
\end{aligned}
$$

Using $q_{2}=1$, we have

$$
\begin{aligned}
\left\|\left({ }_{x} f-f\right) * g\right\|_{\left(s_{1}, \infty\right)}^{\pi} & \leqslant k r^{2} n_{F}(N, V)\left\|_{x} f-f\right\|_{q_{1}, s_{1} ; \infty}^{\pi, E_{0}}\left(\sum_{E^{\prime} \in \pi}\left\|g_{E^{\prime}}\right\|_{q_{1}}\right) \\
& =k r^{2} n_{F}(N, V)\left\|_{x} f-f\right\|_{q_{1}, s_{1} ; \infty}^{\pi, E_{0}}\|g\|_{\underline{q}}^{\pi} .
\end{aligned}
$$

This proves that

$$
\left\|{ }_{x}\left(P_{h} C_{f} g\right)-P_{h} C_{f} g\right\|_{\underline{s}}^{\pi} \leqslant \Gamma(\pi)\left\|_{x} h-h\right\|_{\underline{p}}^{\pi}\left\|_{x} f\right\|_{\underline{r}}^{\pi}+\|h\|_{\underline{p}}^{\pi} k r^{2} n_{F}(N, V)\left\|_{x} f-f\right\|_{q_{1}, s_{1} ; \infty}^{\pi, E_{0}}
$$

The first term on the right approaches 0 as $x$ approaches the identity since we are assuming $p<\bar{\infty}$, the second term does likewise by hypothesis, and the convergence is uniform for $g$ in the unit ball. Thus the image of the unit ball under $P_{h} C_{f}$ satisfies (b) of 3.13 and it clearly satisfies (a) as well.

By (c) of Proposition 3.13, given $\varepsilon>0$ we can choose a compact set $K$ such that $\left\|h_{(G \sim K)}\right\|_{p}^{\pi} \leqslant \varepsilon / \Gamma(\pi)\|f\|_{\underline{r}}^{\pi}$. Then $\left(P_{h} C_{f} g\right)_{G \sim K}=P_{h_{(G \sim K)}}\left(C_{f} g\right)_{G \sim K}$, which has norm bounded by $\left\|h_{(G \sim K)}\right\|_{p}^{\pi} \overline{\Gamma(\pi)}\|f\|_{r}^{\pi}<\varepsilon$, for all $g$ in the unit ball, as required. Thus the image of the unit ball under $P_{h} C_{f}$ is compact if $p_{1}<\infty$.

Consider next $p_{1}=\infty$, so $h$ is in $L_{\left(\infty, p_{2}\right)}^{\pi}$ with $p_{2}<\infty$. For each positive $n$ we can choose Borel $B_{n}$ such that if $h_{n}$ is the restriction of $h$ to the complement of $B_{n}$ then $\left\|h_{n}\right\|_{\left(\infty, p_{2}\right)}^{\pi}<1 / n$. For fixed $n$ we can find $h^{\prime}$ in $K(G)$ identically 1 on $B_{n}$ with range in $[0,1]$. By the previous case, $P_{h^{\prime}} C_{f}$ is compact from $L_{q}^{\pi}$ to $L_{\underline{s}}^{\pi}$, so $\left(P_{h}-P_{h_{n}}\right) C_{f}=$ $\left(P_{h}-P_{h_{n}}\right) P_{h^{\prime}} C_{f}$ is compact and by 5.1, $\left.\left\|P_{h_{n}} C_{f}\right\|_{q, s} \leqslant \Gamma \overline{(} \pi\right)\left\|h_{n}\right\|_{\left(\infty, p_{2}\right)}^{\pi}\|f\|_{\underline{r}}^{\pi} \rightarrow 0$. Thus $P_{h} C_{f}$ is compact which completes the proof of sufficiency.

Before turning to the converse, we note a special case in which the assumption on the indices guarantee the translation-continuity condition, so we can exclude this case in the proof of necessity. Suppose $1 / r_{1}+1 / q_{1}=1+1 / s_{1}$. By hypothesis this implies $p_{1}=\infty$ and, since $s_{1}<\infty, r_{1}<\infty$. We will show for any net $\left\{t_{\alpha}\right\} \rightarrow e$ we have translation-continuity: $\left\|_{t_{\alpha}} f-f\right\|_{q_{1}, s_{i} ; \infty}^{\pi, E_{0}} \rightarrow 0$. If not, there are nets $\left\{t_{\alpha}\right\} \rightarrow e$, $\left\{E_{\alpha}\right\}$ in $\pi, g_{\alpha}$ in $L_{q_{1}}\left(E_{\alpha}\right)$ with $\left\|g_{\alpha}\right\|_{q_{1}}=1$ and $\left\|\left[\left(_{t_{\alpha}} f-f\right) * g_{\alpha}\right]_{E_{0}}\right\|_{s_{1}}>\delta>0$. By 4.2(b) we have $\left\|\left[\left(t_{t_{\alpha}} f-f\right) * g_{\alpha}\right]_{E_{0}}\right\|_{s_{1}} \leqslant C\left\|\left({ }_{t_{\alpha}} f-f\right) * g_{\alpha}\right\|_{\underline{s}}^{\pi}<C \Gamma(\pi)\left\|_{t_{\alpha}} f-f\right\|_{\underline{r}}\left\|g_{\alpha}\right\|_{q}$ $=C \Gamma(\pi)\left\|_{t_{\alpha}} f-f\right\|_{\underline{r}}$ and by 13.5(b) $\left\|_{t_{\alpha}} f-f\right\|_{\underline{r}} \rightarrow 0$ since $\underline{r}<\bar{\infty}$. This contradicts the supposition that $f$ does not satisfy translation-continuity. Thus we can assume $1 / r_{1}+1 / q_{1}-1 / s_{1}<1$ in proving necessity.

Suppose $f$ is not translation-continuous in $L M_{q_{1}, s_{1} ; \infty}^{\pi}$. Since $G$ is first countable there is $\delta>0$ and a sequence $\left\{t_{n}\right\} \rightarrow e$ such that $\left\|_{t_{n}} f-f\right\|_{q_{1}, s_{1} ; \infty}^{\pi, E_{0}}>\delta$. Thus for each $n$ there is $E_{n}$ in $\pi$ such that $\left\|\Lambda_{E_{0}} C_{(f-f)} \Lambda_{E_{n}}\right\|_{q_{1}, s_{1}}>\delta$ for $t=t_{n}$. This means for each $n$ there is $g_{n}$ in the unit ball of $L_{q_{1}}\left(E_{n}\right)$ such that $\left\|\left[\left({ }_{t_{n}} f-f\right) * g_{n}\right]_{E_{0}}\right\|_{s_{1}} \geqslant \delta$. Let 
$k_{n}={ }_{t_{n}}\left(\Lambda_{E_{0}} C_{f} g_{n}\right), p_{n}=\Lambda_{E_{0}} C_{f} g_{n}$. Then

$$
k_{n}-p_{n}=t_{n}\left[\left(\chi_{E_{0}}-\chi_{t_{n} E_{0}}\right) C_{f} g_{n}\right]+\left[\left({ }_{t_{n}} f-f\right) * g_{n}\right]_{E_{0}} .
$$

The above calculations show the norm in $L_{s_{1}}\left(E_{0}\right)$ of the second term exceeds $\delta$, hence so does its norm in $L_{s}{ }^{\pi}$. By continuity of translation (3.13(b)) and the bound of 5.1 for $P C$ operators, the first term is bounded in $L_{s}^{\pi}$ norm by a multiple of $\left\|\chi_{E_{0}}-\chi_{i_{n} E_{0}}\right\|_{\nu} \rightarrow 0$, where $1 / \nu \leqslant 1+1 / s_{1}-1 / r_{1}-1 / q_{1}$. Thus $\left\|k_{n}-p_{n}\right\|_{s}^{\pi}>$ $\delta / 2$ infinitely often, the image of the unit ball under the operator $\Lambda_{E_{0}} C_{f}$ violates 3.13(b) and hence the operator is not compact. Let $A$ be relatively compact, Borel and of positive measure. If $\Lambda_{A} C_{f}$ is compact, so is $\Lambda_{A x^{-1}} C_{f}=T_{x} \Lambda_{A} T_{x^{-1}} C_{f}=$ $T_{x} \Lambda_{A} C_{f} T_{x^{-1}}$ (right translation commutes with left convolution). Since $\Lambda_{A x^{-1}}$ and $\Lambda_{E_{0}}$ commute, all $\Lambda_{A x^{-1}} \Lambda_{E_{0}} C_{f}$ are compact and by 3.13

$$
\begin{aligned}
0 & =\lim _{n \rightarrow \infty}\left\|t_{n}\left(\Lambda_{A x^{-1}} \Lambda_{E_{0}} C_{f} g_{n}\right)-\Lambda_{A x^{-1}} \Lambda_{E_{0}} C_{f} g_{n}\right\|_{\underline{s}}^{\pi} \\
& =\lim _{n \rightarrow \infty}\left\|\Lambda_{A x^{-1}}\left(k_{n}-p_{n}\right)+\left(\Lambda_{t_{n}^{-1} A X^{-1}}-\Lambda_{A X^{-1}}\right)\left[{ }_{t_{n}}\left(\Lambda_{E_{0}} C_{f} g_{n}\right)\right]\right\|_{\underline{s}}^{\pi} .
\end{aligned}
$$

As before, by continuity of translation and the bound for $P C$ operators,

$$
\left\|\left(\Lambda_{t_{n}^{-1} A X^{-1}}-\Lambda_{A x^{-1}}\right)\left[{ }_{t_{n}}\left(\Lambda_{E_{0}} C_{f} g_{n}\right)\right]\right\|_{s}^{\pi} \rightarrow 0
$$

so we must have $\left\|\Lambda_{A x^{-1}}\left(k_{n}-p_{n}\right)\right\|_{\underline{s}}^{\pi} \rightarrow 0$ for all $x$. But

$$
\begin{aligned}
\left\|\Lambda_{A x^{-1}}\left(k_{n}-p_{n}\right)\right\|_{s_{1}} & =\int_{G} \chi_{A x^{-1}}(y)\left|k_{n}(y)-p_{n}(y)\right|^{s_{1}} d m(y) \\
& =\int_{G} \chi_{A}\left(y^{-1}\right)\left|k_{n}\left(y^{-1} x^{-1}\right)-p_{n}\left(y^{-1} x^{-1}\right)\right|^{s_{1}} d m(y) \\
& =\left[\chi_{A^{-1}} *\left|k_{n}-p_{n}\right|^{s_{1}}\right]\left(x^{-1}\right)
\end{aligned}
$$

so this last convolution converges pointwise to zero. By Lebesgue's dominated convergence theorem,

$$
\begin{aligned}
\int_{G}\left[\chi_{A^{-1}} *\left|k_{n}-p_{n}\right|^{s_{1}}\right] & (x) d m(x) \\
& =\int_{G} \int_{G} \chi_{A^{-1}}(y)\left|k_{n}\left(y^{-1} x\right)-p_{n}\left(y^{-1} x\right)\right|^{s_{1}} d m(x) d m(y) \\
& =\int_{G} \chi_{A^{-1}}(y) \int_{G}\left|k_{n}\left(y^{-1} x\right)-p_{n}\left(y^{-1} x\right)\right|^{s_{1}} d m(y) d m(x) \\
& =m\left(A^{-1}\right)\left\|k_{n}-p_{n}\right\|_{s_{1}}^{s_{1}}
\end{aligned}
$$

converges to zero. Since $k_{n}-p_{n}$ has compact support, we have also $\left\|k_{n}-p_{n}\right\|_{s}^{\pi} \rightarrow$ 0 , which contradicts our result that $\left\|k_{n}-p_{n}\right\|_{\underline{s}}^{\pi} \nrightarrow 0$. Thus $\Lambda_{A} C_{f}$ is not compact for any relatively compact Borel $A$ of positive measure. By assumption on $h$ there is such an $A$ on which $|h|>\varepsilon>0$. There is also a bounded measurable $g$ of compact essential support such that $g h=1$ on $A$, so $\Lambda_{A} C_{f}=\left(\Lambda_{A} P_{g}\right) P_{h} C_{f}$. If $\Lambda_{A} C_{f}$ is not compact, $P_{h} C_{f}$ cannot be.

A portion of the above yields 
Corollary 6.10. For $f$ in $L_{\left(r_{1}, \infty\right)}^{\pi}, h$ in $L_{\left(\infty, p_{2}\right)}^{\pi}$ with $r_{1}<\infty, p_{2}<\infty$, the operator $P_{h} C_{f}$ from $L_{\left(q_{1}, 1\right)}^{\pi}$ to $L_{\left(s_{1}, s_{2}\right)}^{\pi}$ is compact if $1 / r_{1}+1 / q_{1}=1+1 / s_{1}>1$, and $p_{2} \leqslant s_{2}<$ $\infty$.

Corollary 6.11. Under the hypotheses of Theorem $\mathrm{B}, P_{h} C_{f}$ is compact whenever $f$ is a translation-continuous vector in $L_{r}{ }^{\pi}$.

Proof. The local multiplier norms of Theorem B are dominated by $r_{1}$ norms. The conditions of 6.11 is often easier to verify than that of Theorem B. Unfortunately it is not necessary. We give a counterexample using the uniform partition of $R$ into the intervals $E_{n}=[2 n \pi, 2(n+1) \pi)$. Let $D_{n}$ be the $n$th Dirichlet kernel:

$$
D_{n}(x)=\frac{\sin \left(n+\frac{1}{2}\right) x}{2 \sin \frac{1}{2} x}
$$

and let $d_{n}$ be the normalized restriction of $D_{|n|}$ to $L_{1}\left(E_{n}\right)$. Let $f=\sum_{n=-\infty}^{\infty} d_{n}$, so $f$ belongs to $L_{(1, \infty)}^{\pi}(R)$.

EXAMPLE 6.12. The function $f$ is translation-continuous in $L M_{2,2 ; \infty}^{\pi}$ (so, e.g., for any $h$ in $L_{(\infty, 1)}^{\pi}, P_{h} C_{f}$ is compact from $L_{(2,1)}^{\pi}$ to $L_{(2,1)}^{\pi}$ but $f$ is not translation-continuous in $L_{(1, \infty)}^{\pi}$.

Proof. For $g$ in $L_{2}\left(E_{k}\right), x$ in $E_{0}$,

$$
\begin{aligned}
(f * g)(x) & =\int_{-\infty}^{\infty} f(y) g(x-y) d y=\int_{-\infty}^{\infty} g(y) f(x-y) d y \\
& =\int_{2 k \pi}^{2(k+1) \pi} f(x-y) g(y) d y \\
& =\int_{2 k \pi}^{x+2 k \pi} d_{k}(x-y) g(y) d y+\int_{x+2 k \pi}^{2(k+1) \pi} d_{k+1}(x-y) g(y) d y .
\end{aligned}
$$

. For $n \geqslant 0, D_{n+1}(x)=D_{n}(x)+\cos (n+1) x$ and the $L_{1}\left(E_{k}\right)$ norms are strictly increasing so, writing the reciprocal of this $L_{1}$ norm of $D_{|k|}$ as $C_{k}$,

$$
\begin{aligned}
|(f * g)(x)| \leqslant & C_{k}\left(\left|\int_{2 k \pi}^{2(k+1) \pi} D_{|k|}(x-y) g(y) d y\right|\right) \\
& +\left|\int_{x+2 k \pi}^{2(k+1) \pi} \cos (|k+1|(x-y)) g(y) d y\right| .
\end{aligned}
$$

As is well known, the first of these integrals is $\pi$ times the projection of $g$ to a subspace of $L^{2}(2 k \pi, 2(k+1) \pi)$, so

$$
\int_{0}^{2 \pi}\left|\int_{2 k \pi}^{2(k+1) \pi} D_{|k|}(x-y) g(y) d y\right|^{2} d x \leqslant \pi^{2} \int_{2 k \pi}^{2(k+1) \pi}|g(y)|^{2} d y .
$$

By direct computation, we also have

$$
\int_{0}^{2 \pi}\left|\int_{x+2 k \pi}^{2(x+1) \pi} \cos (|k+1|(x-y)) g(y) d y\right|^{2} d x \leqslant 4 \pi^{2} \int_{2 k \pi}^{2(k+1) \pi}|g(y)|^{2} d y .
$$

Thus $\int_{0}^{2 \pi}|(f * g)(x)|^{2} d x \leqslant C_{k}^{2} 7 \pi^{2}\|g\|_{2}^{2}$. Since the $C_{k}$ 's converge to zero, $f$ is in $L M_{2,2 ; 0}^{\pi} \subset L M_{2,2 ; \infty}^{\pi}$. Thus given $\varepsilon>0$ we can choose a compact set $K$ such that $\sup _{x}\left\|_{x}\left(1-\Lambda_{K}\right) f\right\|_{2,2 ; \infty}^{\pi, E_{0}}<\varepsilon / 3$. Since $K$ is compact, as $x \rightarrow 0,\left\|_{x}\left(\Lambda_{K} f\right)-\Lambda_{K} f\right\|_{2,2 ; \infty}^{\pi, E_{0}}$ $\leqslant\left\|_{x}\left(\Lambda_{K} f\right)-\Lambda_{K} f\right\|_{(1, \infty)}^{\pi} \rightarrow 0$. Thus if $x$ is small enough $\left\|_{x} f-f\right\|_{2,2 ; \infty}^{\pi, E_{0}}<\varepsilon$, so $f$ is translation-continuous in $L M_{2,2 ; \infty}^{\pi}$. 
For $0<\delta<\pi / 2$, let $E(\delta)=\cup_{n=-\infty}^{\infty}[2 n \pi-\delta, 2 n \pi+\delta)$ and let $F(\delta)$ be its complement. We know

$$
\int_{0}^{2 \pi-\delta}\left|D_{n}(x)\right| d x \leqslant \int_{\delta}^{2 \pi-\delta} \frac{d x}{2 \sin \left(\frac{1}{2} x\right)} \leqslant \frac{2 \pi-2 \delta}{2 \sin (\delta / 2)},
$$

so as $x \rightarrow 0,\left\|_{x}\left(\Lambda_{F(\delta)} f\right)-\Lambda_{F(\delta)} f\right\|_{(1, \infty)}^{\pi} \rightarrow 0$. On the other hand,

$$
\left\|_{2 \delta}\left(\Lambda_{E(\delta)} f\right)-\Lambda_{E(\delta)} f\right\|_{(1, \infty)}^{\pi}=\left\|_{2 \delta}\left(\Lambda_{E(\delta)} f\right)\right\|_{(1, \infty)}^{\pi}+\left\|\Lambda_{E(\delta)} f\right\|_{(1, \infty)}^{\pi}
$$

which can be made arbitrarily close to one. Thus $f$ is not translation-continuous in $L_{(1, \infty)}^{\pi}$.

7. Summary and applications. In this section we review our results, illustrate important special cases, and show how the results can be extended.

Proposition 3.7 establishes that the mixed-norm spaces $L_{(p, q)}^{\pi}(G)$ (for $1 \leqslant p, q \leqslant$ $\infty)$ provide a class of Banach spaces which interpolate (and include isometrically) the usual $L_{p}(G)$ spaces as topological vector spaces, have duality relationships similar to those of the $L_{p}$ spaces, and have the continuous functions of compact support as a dense subset if $p, q<\infty$. Theorem 4.2 provides the analogue of Young's inequality for convolutions on these mixed-norm spaces. (See also [18].) It incidentally establishes that $L_{(p, 1)}^{\pi}(G)$ is always a group algebra and allows us to strengthen a lemma of Stewart [16] to show (for $G$ abelian) there is a bounded $L_{1}$ function on $G$ with Fourier transform of compact support and identically equal to one on a prescribed compact set.

Proposition 5.4 shows the introduction of mixed-norm spaces cannot be avoided, even in studying bounded $P C$ operators on $L_{p}$, since the product and convolution functions must lie in certain mixed-norm spaces but need not lie in any of the usual $L_{p}$ spaces. Theorem 5.1 establishes sufficient conditions for boundedness of $P C$ operators between mixed-norm spaces. Necessary and sufficient conditions for $P_{h} C_{f}$ to be bounded are obtained only for operators from $L_{\infty}$ to itself ( $h$ must be in $L_{\infty}$ and $f$ in $L_{1}$ ). Example 5.6 shows that if the conditions of 5.1 are approximated arbitrarily closely whether the operator is bounded or not can depend on "rate of growth" conditions.

As an application, consider $P C$ operators on $L_{2}(R)$ (which were the original motivation for this study). Let $\pi$ be the partition of $R$ into unit intervals $[n, n+1)$. Proposition 5.4 states that for such an operator $P_{h} C_{f}$ to be bounded it is necessary that $f$ be in $L_{(1,2)}^{\pi}$ and $h$ be in $L_{(2, \infty)}^{\pi}$, i.e. $f$ must be integrable over each interval and the resulting sequence of $L_{1}([n, n+1))$ norms must be square summable while $h$ must be locally square integrable and the sequence of $L_{2}([n, n+1))$ norms must be bounded. Theorem 5.1 says such an operator will be bounded if $f$ is in $L_{\left(r_{1}, r_{2}\right)}^{\pi}$-locally in $L_{r_{1}}([n, n+1))$, with norm sequence in $l_{r_{2}}$-and $h$ is in $L_{\left(p_{1}, p_{2}\right)}^{\pi}$, where $1 / p_{1}+1 / r_{1} \leqslant 1,1 / p_{2}+1 / r_{2} \geqslant 1,2 \leqslant p_{1}, r_{2} \leqslant 2$. (The last two conditions are also necessary, of course.)

Our discussion of compactness is limited to operators $P_{h} C_{f}$ which satisfy the sufficient conditions for boundedness given in Theorem 5.1 acting between spaces where neither index is infinite. In that case Theorem 6.4 shows that all the 
operators for which the "global" second indices of both $h$ and $f$ are finite are compact. For a $P C$ operator in $L^{2}(R)$, for instance, the operator will always be compact unless in the above conditions $p_{2}=\infty$. Theorem A gives necessary and sufficient conditions for compactness of $P_{h} C_{f}$ when the global index of $h$ is infinite. (This, incidentally, answers the question posed in the first paragraph of this paper: if $h$ is in $L_{\infty}(R)$ and $f$ in $L_{1}(R)$ then a necessary and sufficient condition for compactness of $P_{h} C_{f}$ as an operator in $L_{2}(R)$ is that $h$ be in $L_{(1,0)}^{\pi}$, i.e., $h$ must be locally in $L_{1}([n, n+1))$ and the sequence of $L_{1}$ norms must converge to zero. This is equivalent, of course, to the condition given in [2].) The remaining case, when the global index of $f$ is infinite, is dealt with in Theorem B. This can only occur when the global index of the range space is 1 , so does not apply to operators on $L_{2}(R)$. It can be applied to $L_{1}(R)$, however. For instance, Corollary 6.10 shows that $P_{h} C_{f}$ is compact from $L_{1}(R)$ to $L_{1}(R)$ provided $h$ is in $L_{(\infty, 1)}(R)$ (i.e., $h$ is bounded and the local bounds on $[n, n+1)$ are summable) and $f$ is in $L_{(1, \infty)}(R)$ (i.e., $f$ is locally integrable and the $L_{1}$ norms on $[n, n+1)$ are bounded).

To produce a particularly nasty example, let $\theta, \phi$ be in $L_{2}$ with essential support in $[0,1]$ and let

$$
\begin{aligned}
& h(x)=\int_{n=1}^{\infty} \sqrt{n} \theta\left(n x-n^{2}\right), \\
& f(x)=\sum_{n=1}^{\infty} \phi\left(n x-n^{2}\right) / \sqrt{n} \log ^{2}(2 n) .
\end{aligned}
$$

Then $P_{h} C_{f}$ is bounded on $L_{2}(R)$ but not compact. On the other hand, with the same $f$, if $h(x)=\sum_{n=1}^{\infty} \log n \theta\left(n x-n^{2}\right)$ then $P_{h} C_{f}$ is compact. (To be more specific, take $\theta(x)=\phi(x)=1 / \sqrt{x} \log (x / 2)$ on $(0,1]$.)

The class of kernels to which our results apply can be broadened by applying some simple manipulations. By taking adjoints, we obtained results for convolution-product operators $C_{f} P_{h}$ and consideration of $\left(P_{h} C_{f}\right)^{*}\left(P_{h} C_{f}\right)$ yields results for kernels of the form

$$
K(x, t)=\int \bar{f}(x-y)|h(y)|^{2} f(y-t) d y
$$

of ten encountered in applications. A rather different set of kernels can be obtained, however, by using a change of variables. Let $k$ be a monotone increasing absolutely continuous function on $R$ and define $T$ on $L_{2}(R)$ by

$$
(T g)(x)=\int_{-\infty}^{\infty} f(k(x)-y) g(y) d y=-(f * g)(k(x)) .
$$

Then

$$
\begin{aligned}
\left(\|T g\|_{2}\right)^{2} & =\int_{-\infty}^{\infty}|(f * g)(k(x))|^{2} d x \\
& =\int_{-\infty}^{\infty}|(f * g)(k)|^{2}\left(\frac{d x}{d k}\right) d k=\left(\left\|P_{h} C_{f} g\right\|_{2}\right)^{2}
\end{aligned}
$$

where $h=(d x / d k)^{1 / 2}$. Thus boundedness of $T$ reduces to that of a $P C$ operator. Moreover there are clearly partial isometries $U$ and $V$ such that $P_{h} C_{f}=U T$, $T=V P_{h} C_{f}$, so $T$ is compact iff $P_{h} C_{f}$ is. Similarly, if we let 


$$
(S g)(x)=\int_{-\infty}^{\infty} f(x-k(y)) g(y) d y
$$

we need only observe that $S$ is the adjoint of an operator of the type $T$ defined previously (with $f$ replaced by $\bar{f}^{\sim}$ ). Its boundedness and compactness, therefore, are also determined by those of a $P C$ operator, namely $\boldsymbol{P}_{h} C_{\bar{f}^{-}}$. Thus our results embrace kernels of the type $f(k(x)-y)$ and $f(x-k(y))$ as well as those of the form $h(x) f(x-y)$ and their adjoints $f(x-y) h(y)$. Obviously, the restrictions to monotone $k$ in the above discussion is overly stringent and can be weakened to demand only that the induced measure be absolutely continuous. The $d x / d k$ in the above calculation then becomes the Radon-Nikodym derivative.

For specific examples, let $f$ be in $L_{1}(R)$ and define the operators $T_{1}, T_{2}, S_{1}$ and $S_{2}$ on $L_{2}(R)$ by

$$
\begin{aligned}
& \left(T_{1} g\right)(x)=\int_{-\infty}^{\infty} f\left(e^{|x|}+\sin |x|-y\right) g(y) d y, \\
& \left(S_{1} g\right)(x)=\int_{-\infty}^{\infty} f\left(x-e^{|y|}-\sin |y|\right) g(y) d y, \\
& \left(T_{2} g\right)(x)=\int_{-\infty}^{\infty} f(|x|+\log |x|-y) g(y) d y \\
& \left(S_{2} g\right)(x)=\int_{-\infty}^{\infty} f(x-|y|-\log |y|) g(y) d y .
\end{aligned}
$$

Application of our results as outlined above then shows that $T_{1}$ and $S_{1}$ are compact while $T_{2}$ and $S_{2}$ are bounded but not compact.

8. Acknowledgements. The authors are indebted to Professor Jesus Gil de Lamadrid for bringing reference [18] to their attention and to Professor Loren Argabright for numerous discussions including the observation that the functions considered in 3.9 provide new equivalent norms.

ADDED IN PROOF. The authors have recently been made aware of several publications by H. G. Feichtinger which relate to mixed norm spaces as discussed in this paper. See, for example, [19] and [20].

\section{REFERENCES}

1. A. Benedek and R. Panzone, The spaces $L^{p}$ with mixed norm, Duke Math. J. 28 (1961), 301-324. MR 23A \#3451.

2. R. C. Busby, I. Schochetman and H. A. Smith, Integral operators and the compactness of induced representations, Trans. Amer. Math. Soc. 164 (1972), 461-477. MR 45 \#4167.

3. R. C. Busby and I. Schochetman, Compact induced representations, Canad. J. Math. 24 (1972), 5-16. MR 45 \#2495.

4. N. Dunford and J. T. Schwartz, Linear operators. I, Interscience, New York, 1958.

5. W. R. Emerson and F. P. Greenleaf, Covering properties and Folner conditions, Math. Z. 102 (1967), 370-384. MR 36 \#3912.

6. S. Grosser and M. Moskowitz, Compactness conditions in topological groups, J. Reine. Angew Math. 246 (1971), 1-40. MR 44 \#1766.

7. E. Hewitt and K. A. Ross, Abstract harmonic analysis. I, Academic Press, New York, 1963. MR 28 \# 158.

8. F. Holland, Harmonic analysis on amalgams of $L^{p}$ and $l^{q}$, J. London Math. Soc. (2) 10 (1975), 295-305. MR 51 \# 11013.

9.

On the representation of functions as Fourier transforms of unbounded measures, Proc. London Math. Soc. (3) 30 (1975), 347-365. 
10. C. N. Kellogg, An extension of the Hausdorff-Young theorem, Michigan Math. J. 18 (1971), 121-127. MR 43 \#6714.

11. K. Knopp, Infinite sequences and series, Dover, New York, 1956. MR 18, 30.

12. W. A. J. Luxemburg and A. C. Zaanen, Compactness of integral operators in Banach function spaces, Math. Ann. 149 (1962/63), 150-180. MR 26 \#2905.

13. R. Mosak, Central functions in group algebras, Proc. Amer. Math. Soc. 29 (1971), 613-616. MR 43 \#5323.

14. N. Rickert, Convolution of $L^{p}$ functions, Proc. Amer. Math. Soc. 18 (1967), 762-763. MR 35 \#7136.

15. W. Rudin, Functional analysis, McGraw-Hill, New York, 1973. MR 51 \#1315.

16. J. Steward, Unbounded positive definite functions, Canad. J. Math. 21 (1969), 1309-1318. MR 40 \#4689.

17. L. Williams, Generalized Hausdorff-Young inequalities and mixed norm spaces, Pacific J. Math. 38 (1971), 823-833. MR 46 \#9653.

18. J.-P. Bertrandias, C. Datry and C. Depuis, Unions et intersections d'espaces $L^{p}$ invariantes par translation ou convolution, Ann. Inst. Fourier (Grenoble) 28 (1978), 53-84.

19. H. G. Feichtinger, On a class of convolution algebras of functions, Ann. Inst. Fourier (Grenoble) 27 (1977), 135-162.

20. __ Banach convolution algebras of functions. II, Mh. Math. 87 (1979), 181-207.

Department of Mathematics, Drexel University, Philadelphia, Pennsylvania 19100

Department of Mathematics, Arizona State University, Tempe, Arizona 85281 